
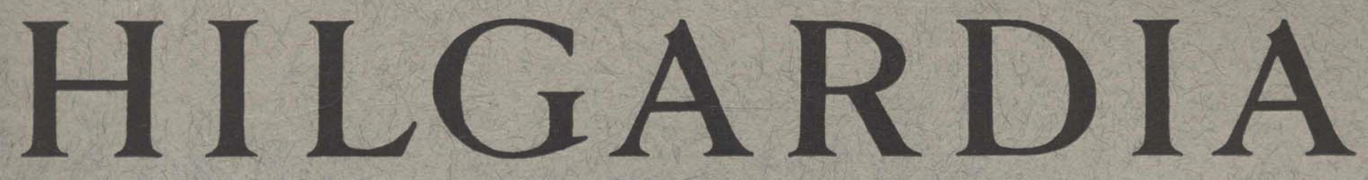

A Journal of Agricultural Science Published by the California Agricultural Experiment Station

\title{
THE PHLOEM OF THE SWEET ORANGE TREE TRUNK AND THE SEASONAL PRODUCTION OF XYLEM AND PHLOEM
}

\author{
HENRY SCHNEIDER
}

UNIVERSITY OF CALIFORNIA • BERKELEY, CALIFORNIA 
This paper is a report of an anatomical research undertaken in 1947 on the trunks of mature sweet orange trees on sweet orange rootstock. Object of the investigation was to defermine the time at which new trunk phloem is produced, with respect to the time of shoot and root extension growth and xylem production. Also investigated were the anatomic features of xylem and phloem development; the portion of the phloem which is replaced during the year; and how and when the older sieve tubes degenerate.

Appended to the present report is a detailed method for collecting, sectioning, and staining large batches of citrus phloem.

Table of Contents is on outside back cover. 


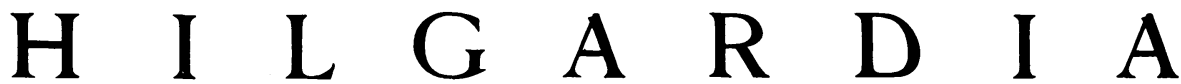

A Journal of Agricultural Science Published by

the California Agricultural Experiment Station

VoL. 21

APRIL, 1952

No. 12

\section{THE PHLOEM OF THE SWEET ORANGE TREE TRUNK AND THE SEASONAL PRODUCTION OF XYLEM AND PHLOEM ${ }^{1,2}$}

\author{
HENRY SCHNEIDER ${ }^{3}$
}

\section{INTRODUCTION}

The primary purpose of this investigation was to determine for the trunks of sweet orange trees (Citrus sinensis Linn.): (1) the period during the growing season, with relation to shoot, root, and xylem growth, when new phloem was produced, (2) how long annual increments of phloem function, and (3) what time of the year degeneration of the older sieve tubes occurs. The study was undertaken in connection with an anatomical study of trees affected by orange tree quick decline (Schneider, 1946). ${ }^{4}$ Orange tree quick decline is a virus disease newly introduced into southern California (Wallace and Fawcett, 1947) which causes sieve tube necrosis in the sour orange stock (C. aurantium Linn.) of sweet orange trees (Schneider, 1946). Webber and Fawcett (1935) have described the functioning phloem of the sweet orange, but its development and degeneration have not been considered. Because of the variable effects which different varieties of sour orange used as rootstock have upon the phloem of the scion, only sweet orange trees on sweet orange stock are considered in this paper. Another paper will describe in less detail the phloem of the sour orange, that of the trifoliate orange (Poncirus trifoliata Linn.), and the phloem of the sour orange when used as a stock under sweet orange.

\section{REVIEW OF LITERATURE}

It may be concluded from the literature that there are three periods of shoot extension growth in sweet orange trees when growing in a climatic condition such as that of southern California (Reed and MacDougal, 1937;

${ }^{1}$ Manuscript submitted for publication April 19, 1951.

2 Paper No. 713, University of California Citrus Experiment Station, Riverside California.

- Assistant Plant Pathologist in the Experiment Station, Riverside.

"See "Literature Cited" for citations, referred to in the text by author and date. 
Cameron and Schroeder, 1945). The time of these flushes varies from year to year and from orchard to orchard, and their duration varies with the size of the trees. The flushes are referred to as the spring, summer, and autumn flushes.

In 1927 Crider working in Arizona reported the discovery of the phenomenon that three cycles of extension growth of shoots alternated with three cycles of extension growth of roots in citrus species less than four years old. His work indicated that an extended dormant period occurred in the winter during which there was neither shoot nor root growth. Shoot growth preceded root growth in the spring. The alternation of shoot and root growth in older bearing trees was not so marked. Waynick and Walker (1930), working in the coastal area of California with several species of trees of various ages budded on sour orange stock, confirmed these results to some extent. Waynick and Walker's report is unclear as to the age and species of plants for which they are describing root growth. Apparently referring to old Valencias, they found that roots in the subsoil grew before the top growth commenced during one year and also that the cycle of root growth which followed the second cycle of top growth overlapped the entire third cycle of top growth. There was little root growth after the third cycle of top growth. Reed and McDougal (1937), working in the Riverside district of California with navel oranges six to eight feet tall on sweet orange stock, found two cycles of root growth which alternated with three cycles of top growth. Top growth occurred before root growth at the beginning of the season.

Radial growth. The problem of detecting radial growth in trunks of trees is difficult because changes in diameter result from causes other than growth and because cambial activity is irregular around the tree (Lodewick, 1928; Priestley, 1930). The most important cause of diametral changes other than growth appears to be related to the water tension within the tree, which in turn is related to relative humidity, temperature, air movement, and soil moisture (Haasis, 1934). Water on the outside of trunks is said to cause swelling (Lodewick, 1928). Tree shrinkage has also been found to accompany dormancy (Haasis, 1934). Daubenmire and Deters (1947) noted a 30 per cent shrinkage in the annual growth increment of Robinia which took place abruptly after the grand period of growth. The most sensitive method for detecting diametral changes is by the use of dendrographs and dendrometers (MacDougal, 1936). Some workers have objected to their use for growth measurements because they measure the growth of only a small point on the tree and because, as mentioned above, growth is only one cause of diametral change (Lodewick, 1928; Priestley, 1930). Lodewick concluded that the measurement of new xylem in sections was the only accurate method for determining the radial growth which occurs over short periods of time, but he realized that the width of annual rings is variable around the tree.

Early workers made extensive investigations with regard to where radial growth begins on trees in the spring, and considerable discrepancy exists. Priestley $(1930,1935)$, in reviewing the literature on the subject and in reporting further investigations, has concluded that for hardwood trees growing in England the beginning of cambial activity is associated with the beginning of extension growth of buds. In trees with diffuse-porous wood the resumption 
of radial growth is slower than in those with the ring-porous xylem. Moreover, in trees with diffuse-porous wood cambial reactivation extends very gradually basipetally into the larger limbs and trunk, whereas in trees with ring-porous wood it spreads down the tree rapidly as soon as the buds swell. The resumption of radial growth was very irregular at points around trunks because growth began below adventitious buds. Possibly the growth in the trunks in advance of that in the limbs observed by other workers was due to growth below adventitious buds. This theory of Priestley's, for the resumption of radial growth in trees with diffuse-porous wood, seems to apply to the orange tree.

Cameron and Schroeder (1945) found that shortly after the orange tree initiated its first flush of shoot growth in the spring, cambial activity began on twigs at the point of the beginning of extension growth and then spread basipetally into the larger limbs and trunk of the tree, and eventually into the roots. In some twigs cambial activity did not start until the extension growth had ceased. Irregularity in the beginning of cambial activity was noted in orange tree trunks. Cambial activity was of short duration in the twigs but was renewed later during subsequent growth flushes. The cambial activity in the trunks and scaffold branches did not seem to be much influenced by the later cycles of growth of twigs and roots.

Chowdhury and Tandan (1950) report that in the broadleaf trees of India, extension growth of the shoots precedes radial growth by from two weeks to three months, irrespective of whether they are diffuse- or ringporous. Apparently the seasonal activity of the cambium for different climates and for different kinds of trees (those with ring-porous or diffuse-porous wood, evergreens, deciduous) should be correlated with the conditions of the phloem (i.e., as to whether it is functioning or nonfunctioning) at the time of cambial awakening. Some phloem would no doubt be necessary to carry carbohydrates into growing shoots. Also cambial awakening should be considered in relation to growing buds and not to whether it first begins in the bole, limbs, and twigs.

There seems to be little doubt that the first shoot growth of orange trees stimulates cambial activity which gradually extends basipetally into the trunks. Once trunk radial growth has begun, the possibilities of the occurrence of (1) growth and rest periods therein, (2) a division of labor of the cambium in producing exclusively xylem or phloem, or (3) variations in cell types produced at different times in the xylem and phloem are of interest. Cameron and Schroeder (1945) were of the opinion that growth cycles of the shoots of orange trees have no effect on cambial activity in the scaffold branches and in the trunk. Reed and MeDougal (1937), using the questionable dendrometer method for measuring orange tree trunk growth, reported that there was a strong tendency toward the alternation of shoot growth and trunk cambial activity. Their growth-rate curve is plotted irregularly as to time, and the alternation of the cycles of shoot-growth flushes with periods of radial growth is far from clear cut. Lodewick (1928) reports that in deciduous ringporous trees there is a rest period between the production of spring and summer wood. At the time of the rest period fibers and sclereids first form in the phloem. 
In reviewing the literature on the relative time of xylem and phloem production, Esau (1948) found the following patterns reported: "(1) that the formation of xylem begins before that of the phloem; (2) that the formation of xylem and that of phloem begin and cease at the same time; (3) that phloem initiation follows that of the xylem and lasts longer; (4) that more cells are cut off on the xylem than on the phloem side." The validity of these patterns was laid open to question "because writers usually make no clear distinction between the maturation of cells left over from the cambial activity of the previous season and the differentiation of cells formed by the new growth."

Wood and bark of the sweet orange. Webber and Fawcett (1935) have described the wood of the sweet orange as follows: "The Citrus sinensis wood studied is pale yellow, diffuse porous but with visible growth rings, hard, and of fine texture, the pores and rays being barely visible without a lens. With the aid of a lens the pores are seen to be fairly uniformly distributed throughout a growth ring, chiefly solitary or in radial multiples of two to five, but occasionally in irregular or circular clusters of three to five. Wood parenchyma is moderately abundant, chiefly paratracheal and metatracheal, but partly diffuse. Libriform wood fibers are the dominant element of the wood. Rays are commonly narrower than the pores, straight or somewhat curved, and light colored, their distance apart being one to three pore widths." Minute structure of the various elements is also described. They observed that diffuse parenchyma strands were composed of four to eight nearly cubical cells, each of which contained a calcium oxalate crystal.

The bark of the orange tree has also been described by Webber and Fawcett (1935). With regard to the phloem they report: "In older stems, bands of phloem fibers alternate with bands composed of the other phloem elements. The rings of both thick- and thin-walled elements are broken by the phloem rays." The thin-walled areas contain parenchyma cells, crystal idioblasts, sieve tubes, and companion cells. Other details are also presented.

\section{MATERIALS AND METHODS}

Ten 30-year-old Valencia orange trees budded on sweet orange rootstock were chosen for the study. They were interplanted between grapefruit trees, and were in two nonadjacent rows serving as buffer rows between fertilizer test plots at the Citrus Experiment Station at Riverside. The various observations and collections were made every ten days during the 1947 experimental period.

The occurrence of extension growth of the shoots was determined by merely observing when the shoots were or were not growing. Likewise the extension growth of roots was determined by observing whether or not new white tips were present. A trench about a foot deep was dug and a window glass was placed over one side of it in such a way that feeder roots were near the glass. Loose soil was placed against the observation side of the glass between readings. Observations were also made by digging up a few feeder roots.

The occurrence of radial growth was determined by (1) circumference measurements of the trunk with a steel tape, (2) stage of maturity of the xylem mother cells, and (3) the point of tearing of the bark from the wood. 
After July 28, all trunk measurements were made at about 8:00 A.M. in order to avoid the shrinkage which occurred during the day. Since radial growth is usually presented as increases in diameter, the circumference measurements were converted to diameters. As an aid in detecting the degree of maturity of the xylem mother cells, the sections were mounted in a solution of phloroglucinol in 18 per cent hydrochloric acid in order to determine whether lignification had begun.

Samples of wood and bark were taken from five of the trees every ten days. The trees were paired according to their positions in the two rows and one tree of each pair was sampled at intervals of about one inch apart around the trunk for the first half of the year. During the second half of the year the trunk of the other tree of each pair was sampled. The north side of the trees was not sampled because north winds caused some defoliation there. Since Calavan (1947) found that growth is much more pronounced on the southeast side of lemon (Citrus limonia) trunks than elsewhere, sampling was begun on the east side of some of the trees and continued clockwise. On other trees, sampling was started from the south side and continued clockwise. By this method part of the trees were being sampled on the southeast and part on the southwest on each collection date.

The wood and bark samples were fixed in Randolph's modified Navashin's solution, sectioned on a freezing microtome stained progressively with Heidenhain's haematoxylin, counterstained with lacmoid and mounted in Canada balsam. A detailed description of methods for handling large numbers of batches of material, and a staining method for citrus phloem are presented in the Appendix (p. 352).

\section{OBSERVATIONS DURING 1947}

\section{Periodicity of Extension Growth of Shoots and Roots and Radial Growth of Trunks}

The time of occurrence of extension growth of shoots and roots, and the radial growth of trunks for 1947 are presented in relation to each other in figure $1, A$ and $B$. Of the types of growth considered in this paper, shoot extension growth was the first to be observed in the spring of 1947 (figure $1, A)$. The time of occurrence of growth in all of the trees was quite uniform. The first flush of top growth was a short one in which a high percentage of the buds began to grow and in which most shoots were terminated by blossoms when they were only a few inches long. There were two other subsequent growth periods, one in the summer and one in the autumn in which only occasional vegetative shoots were growing at any one time. The shoots attained a greater length and leaves grew larger than in the spring flush.

Although one complete root-growth stoppage was observed during the growing season, there was a definite overlapping of the root-growth periods with the second and third flushes of top growth (figure 1, $A$ ). The quantities of root growth observed on sampling dates during the two growth periods were quite variable, as was also the amount of growth in various samples of feeder roots from the same or different trees. Unfortunately the methods used were designed to determine when root growth was occurring (figure 1, A) 
and the quantities of growth were merely observed in a rough way. Roots failed to contact the glass windows in several cases, air pockets formed under some of the windows and various bugs inhabited the area under the glass. Therefore, observations through the windows were gradually supplemented by digging additional roots. The early observations were rather sketchy because of the faulty methods and because the author was under the impres-

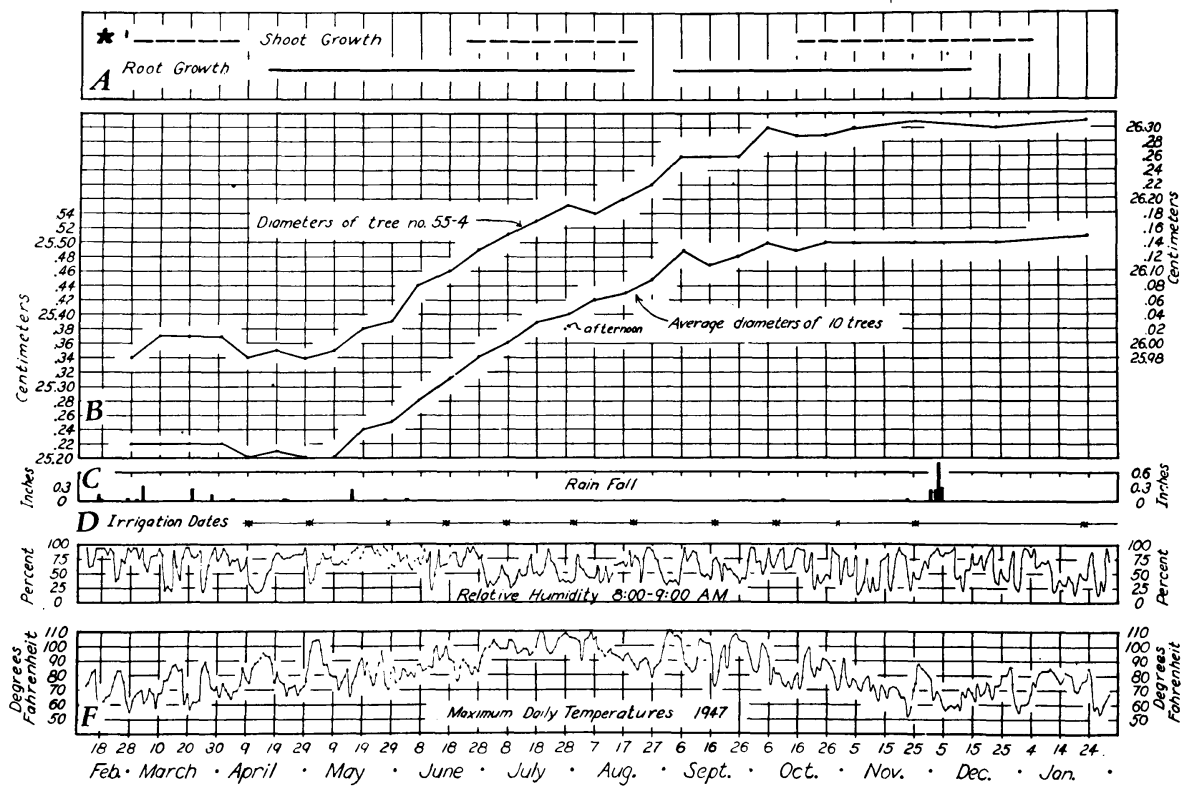

Fig. 1. Graphs of the various types of growth occurring in ten Valencia orange trees, the dates on which irrigations were made, and weather conditions, 1947. A: Broken lines indicate when extension growth of shoots was occurring; solid lines indicate extension growth of roots. $B$, diameters of trees as computed from circumference measurements. $C$, amounts of rainfall. $D$, dates on which irrigations were made. $E$, relative humidity. $F$, maximum daily temperatures.

sion that the phenomenon of alternation of shoot and root growth was well established. It can definitely be stated that abundant root growth was observed under all of the trees at times when flushes of shoot growth had been occurring for some time. After an unusual amount of digging on August 27 no growing roots were found. Ten days later on September 6 abundant growth was present. It is of interest that abundant root growth was observed on September 6 and 16 when the soil was the driest of any time during the year, the trees were under the greatest moisture stress because of high temperatures and low humidities, and radial growth in the trunks had stopped (figure $1 A-F^{\prime}$ ). In January of 1948, at a time when buds were just beginning to grow, occasional root growth was observed under one of two trees when trenches three feet long and four feet deep were dug.

In summary, two cycles of root growth occurred which completely overlapped the cycles of shoot extension growth.

Slippage. A study was made of where slipping of the bark from the wood

* New growth about one inch long was present when observations began. 
occurred at various times during the year. From about October to May the cambium lost its identity and there was a zone of undifferentiated cells several layers wide between the bark and the wood (plate 1). During this period slippage occurred at times and the bark broke loose either from the face of the lignified wood (plate 2, $C$ ), from the face of the phloem (plate $2, B$ ), or at various places through the cambial zone (plate $2, A$ ). When cambial activity began about May 9 (May 19 for one tree), slippage was entirely in the initiating layer of cells at the middle of the cambial zone (plate 2, $D$ ). After new xylem had been produced slippage occurred either at the cambium or in the xylem mother cells (plate $2, E$ ). On July 28 , September 6 , and September 16 , slippage on several of the trees was entirely through the xylem mother cells (plate 5, B) and did not occur as readily as on other dates. In some instances on these dates slippage was by separation at the middle lamella of elongating fibers. The complete absence of slipping in the cambium was taken to indicate a temporary halt in cambial activity.

Trunk growth. The graphs in figure $1, B$, show changes in trunk diameter for one of the trees and for an average of ten of them. As previously mentioned, a number of factors besides growth may affect changes in diameter. It was noted that the average circumference measurements for ten trees was $0.2 \mathrm{~mm}$ less on the hot afternoon of July 28 than on the following morning (figure 1, B). In April there was a slight shrinkage of the trunks and during the same period in collecting bark samples, it was noted that the bark broke loose from the wood in only half as many trees as in late February and early March. Perhaps there was a shrinkage of the cambial region as the cell walls became tougher.

Growth began on May 9 and continued at a uniform rate of about $0.3 \mathrm{~mm}$ per ten-day period through July 18, after which the grand period of growth seemed to be ended and a slower erratic growth commenced. There was a change in the slope of the growth curve after July 18. On July 28 the following changes in growth were observed:

(1) A cessation of cambial activity in two of the trees was indicated by slippage occurring less readily, and by its occurring entirely through the xylem mother cells. This was the first time during the season that slippage was not occurring partly in the cambium. In one of these trees the cells had come apart at the middle lamella rather than by breakage of the xylem mother cell walls. In one tree the bark and wood had failed to slip, but injury to the bark was indicated by degeneration of the functioning sieve tubes in half of the section.

(2) Sieve tube formation gave way to the formation of extensive bands of phloem fiber initials for the first time during the season (plate $5, B$, and table 1, pp. 338-343).

(3) In the xylem, metatracheal parenchyma which had been absent earlier began to appear in small amounts about June 28, and by August 7 long bands were present. Radial growth was slower and erratic for the remainder of the season.

The possible effects of soil moisture, atmospheric humidity and air temperature on radial growth are of interest. Xylem and phloem formation which proceeded at a uniform rate through July 18 had the above-mentioned 
TABLE 1. THE OCCURRENCE OF SLIME BODIES AND FIBER INITIALS IN THE PHLOEM AND OF IMMATURE XYLEM AT VARIOUS TIMES OF THE YEAR, FEBRUARY 1947-JANUARY 1948.

Tree PaIr I

\begin{tabular}{|c|c|c|c|c|c|}
\hline \multirow[t]{2}{*}{$\begin{array}{l}\text { (From Feb. 1947 } \\
\text { to Jan. 1948) }\end{array}$} & \multirow[t]{2}{*}{$\begin{array}{l}\text { Side of tree } \\
\text { sampled }\end{array}$} & $\begin{array}{l}\text { Number of } \\
\text { slime bodies } \\
\text { present in } \\
4 \text { sections }\end{array}$ & $\begin{array}{c}\text { Length of } \\
\text { tangential } \\
\text { bands of } \\
\text { fiber initials }\end{array}$ & $\begin{array}{l}\text { Estimated } \\
\text { amount of } \\
\text { secondary wall } \\
\text { deposited on } \\
\text { fiber initials }\end{array}$ & $\begin{array}{c}\text { Radial } \\
\text { width of new } \\
\text { unlignified } \\
\text { xylem }\end{array}$ \\
\hline & & number & $\mu$ & $\%$ of total & $\mu$ \\
\hline
\end{tabular}

Tree I

\begin{tabular}{|c|c|c|c|c|c|}
\hline Feb. $28 \ldots \ldots \ldots \ldots \ldots$ & $\mathbf{E}$ & 0 & 600 & $30-80$ & 0 \\
\hline Mar. $10 \ldots \ldots \ldots \ldots$ & $\downarrow$ & 0 & 0 & $\ldots \ldots$ & 0 \\
\hline Mar. $19 \ldots \ldots \ldots \ldots$ & $\downarrow$ & 0 & 0 & $\ldots$ & 0 \\
\hline April $1 \ldots \ldots \ldots \ldots$ & $\downarrow$ & 0 & 0 & $\ldots$ & 0 \\
\hline April $9 \ldots$ & SE & 0 & 0 & $\ldots$ & 0 \\
\hline April $19 \ldots \ldots \ldots \ldots$ & $\downarrow$ & 0 & 70 & $10-90$ & 0 \\
\hline April $29 \ldots \ldots \ldots \ldots$ & $\downarrow$ & 1 & 0 & $\ldots \ldots$ & 0 \\
\hline May 9 . & $\downarrow$ & 1 & 0 & $\ldots$ & 0 \\
\hline May $19 \ldots$. & $\mathbf{S}$ & 0 & 0 & $\ldots \ldots$ & 110 \\
\hline May $29 \ldots \ldots \ldots \ldots$ & $\downarrow$ & 0 & 0 & $\ldots$ & 160 \\
\hline June $7 \ldots \ldots \ldots \ldots$ & $\downarrow$ & 1 & 0 & $\ldots$ & 150 \\
\hline June 20. & SW & 7 & 0 & $\ldots \ldots$ & 210 \\
\hline June 28. & $\downarrow$ & 21 & 0 & $\ldots \ldots$ & 210 \\
\hline July $8 \ldots$ & $\downarrow$ & 16 & 0 & $\ldots \ldots$ & 210 \\
\hline July $18 \ldots \ldots$ & $\downarrow$ & 9 & 0 & $\ldots \ldots$ & 210 \\
\hline July $28 \ldots \ldots \ldots$ & W & 10 & 300 & 0 & 180 \\
\hline
\end{tabular}

Tree II

\begin{tabular}{|c|c|c|c|c|c|}
\hline Aug. $\quad 7 \ldots \ldots \ldots \ldots \ldots$ & $\mathbf{S}$ & 2 & 1600 & $\mathbf{0}$ & 80 \\
\hline Aug. $18 \ldots \ldots \ldots \ldots \ldots$ & $\downarrow$ & 1 & 1200 & $\mathbf{0}$ & 50 \\
\hline Aug. $27 \ldots$ & $\downarrow$ & . & $\ldots$ & $\ldots \ldots$ & $\ldots$ \\
\hline Sept. $6 \ldots \ldots$ & SW & 0 & 0 & $\ldots \ldots$ & 125 \\
\hline Sept. $16 \ldots \ldots \ldots \ldots \ldots$ & $\downarrow$ & 0 & 0 & $\ldots \ldots$ & 40 \\
\hline Sept. 26............ & $\downarrow$ & 0 & 850 & 0-30 & 0 \\
\hline Oct. $\cdot 6 \ldots$ & $\mathbf{W}$ & 0 & 1600 & 0-30 & 0 \\
\hline Oct. 16. & $\mathbf{E}$ & 2 & 2500 & $30-90$ & 130 \\
\hline Oct. 27 . & $\downarrow$ & 4 & 0 & $\ldots \ldots$ & 75 \\
\hline Nov. $5 \ldots$ & SE & 0 & 1400 & $0-30$ & 75 \\
\hline Nov. $26 \ldots .$. & $\downarrow$ & 0 & 1500 & $0-40$ & 25 \\
\hline Dec. $24 \ldots$ & $\downarrow$ & 0 & 0 & $\ldots$ & 0 \\
\hline Jan. 26 . & $\mathbf{S}$ & 0 & 0 & $\ldots \ldots$ & 0 \\
\hline
\end{tabular}


TABLE 1-Continued

Tree PaIr II

\begin{tabular}{|c|c|c|c|c|c|}
\hline \multirow[t]{2}{*}{$\begin{array}{l}\text { (From Feb. } 1947 \\
\text { to Jan. 1948) }\end{array}$} & \multirow[t]{2}{*}{$\begin{array}{l}\text { Side of tree } \\
\text { sampled }\end{array}$} & $\begin{array}{l}\text { Number of } \\
\text { slime bodies } \\
\text { present in } \\
4 \text { sections }\end{array}$ & $\begin{array}{c}\text { Length of } \\
\text { tangential } \\
\text { bands of } \\
\text { fiber initials }\end{array}$ & $\begin{array}{l}\text { Estimated } \\
\text { amount of } \\
\text { secondary wall } \\
\text { deposited on } \\
\text { fiber initials }\end{array}$ & $\begin{array}{c}\text { Radial } \\
\text { width of new } \\
\text { unlignified } \\
\text { xylem }\end{array}$ \\
\hline & & number & $\mu$ & $\%$ of total & $\mu$ \\
\hline
\end{tabular}

Tree I

\begin{tabular}{|c|c|c|c|c|c|}
\hline Feb. $28 \ldots \ldots \ldots \ldots \ldots$ & $\mathbf{S}$ & 0 & 0 & $\ldots \ldots$ & 0 \\
\hline Mar. 10 . & $\downarrow$ & 0 & 160 & $30-90$ & 0 \\
\hline Mar. 19. & $\downarrow$ & 0 & 0 & $\ldots \ldots$ & 0 \\
\hline April $1 \ldots \ldots \ldots \ldots \ldots$ & SW & 0 & 0 & $\ldots \ldots$ & 0 \\
\hline Apr. 9 . & $\downarrow$ & 0 & 0 & $\ldots \ldots$ & 0 \\
\hline Apr. $19 \ldots \ldots$ & $\downarrow$ & 0 & 0 & $\ldots \ldots$ & 0 \\
\hline Apr. 29. & $\downarrow$ & 0 & 0 & $\ldots \ldots$ & 0 \\
\hline May $9 \ldots$ & $\mathbf{W}$ & 1 & $\mathbf{0}$ & $\ldots \ldots$ & 0 \\
\hline May $19 \ldots \ldots \ldots \ldots$ & $\mathbf{E}$ & 2 & 0 & $\ldots \ldots$ & 125 \\
\hline May $29 \ldots \ldots \ldots \ldots$ & $\downarrow$ & 1 & 0 & $\ldots \ldots$ & 210 \\
\hline June 7. & $\downarrow$ & 0 & 0 & $\ldots \ldots$ & 180 \\
\hline June $20 \ldots$ & SE & 24 & 0 & $\ldots \ldots$ & 160 \\
\hline June 28... & $\downarrow$ & 26 & 0 & $\ldots \ldots$ & 125 \\
\hline July $\quad 8 \ldots \ldots \ldots$ & $\downarrow$ & 20 & 210 & 0 & 160 \\
\hline July $18 \ldots \ldots \ldots \ldots$ & $\downarrow$ & 4 & 0 & $\ldots \ldots$ & 200 \\
\hline July $28 \ldots \ldots \ldots \ldots$ & $\mathbf{S}$ & 0 & 3500 & 0 & 150 \\
\hline
\end{tabular}

Tree II

\begin{tabular}{|c|c|c|c|c|c|}
\hline Aug. $\quad 7 \ldots \ldots \ldots \ldots \ldots$ & $\mathbf{E}$ & 3 & 2500 & 0 & 140 \\
\hline Aug. $18 \ldots \ldots \ldots \ldots \ldots$ & $\downarrow$ & 0 & 3200 & 0 & 160 \\
\hline Aug. 27... & $\downarrow$ & $\mathbf{0}$ & 0 & $\ldots \ldots$ & 80 \\
\hline Sept. $6 \ldots . .$. & SE & $\mathbf{0}$ & 1000 & 0 & 110 \\
\hline Sept. $16 \ldots \ldots \ldots \ldots \ldots$ & $\downarrow$ & 1 & 500 & 0 & 0 \\
\hline Sept. $26 \ldots . . \ldots$ & $\downarrow$ & 2 & 0 & $\ldots \ldots$ & 110 \\
\hline Oct. $6 \ldots$ & $\mathbf{S}$ & 3 & 0 & $\ldots \ldots$ & 130 \\
\hline Oct. 16. . & $\downarrow$ & $\mathbf{0}$ & 0 & $\ldots \ldots$ & 0 \\
\hline Oct. $27 \ldots \ldots$ & $t$ & 0 & 0 & $\ldots \ldots$ & 160 \\
\hline Nov. $\quad 5 \ldots . .$. & SW & 7 & 0 & $\ldots \ldots$ & 110 \\
\hline Nov. $26 \ldots .$. & $\downarrow$ & 0 & 0 & $\ldots \ldots$ & 60 \\
\hline Dec. $24 \ldots$ & $\downarrow$ & 0 & 2300 & $30-90$ & 0 \\
\hline Jan. $26 \ldots$ & W & 0 & 0 & $\ldots \ldots$ & 0 \\
\hline
\end{tabular}


TABLE 1-Continued

Tree Pair III

\begin{tabular}{|c|c|c|c|c|c|}
\hline \multirow[t]{2}{*}{$\begin{array}{l}\text { (From Feb. } 1947 \\
\text { to Jan. 1948) }\end{array}$} & \multirow[t]{2}{*}{$\begin{array}{l}\text { Side of tree } \\
\text { sampled }\end{array}$} & $\begin{array}{l}\text { Number of } \\
\text { slime bodies } \\
\text { present in } \\
4 \text { sections }\end{array}$ & $\begin{array}{c}\text { Length of } \\
\text { tangential } \\
\text { bands of } \\
\text { fiber initials }\end{array}$ & \begin{tabular}{|l|} 
Estimated \\
amount of \\
secondary wall \\
deposited on \\
fiber initials
\end{tabular} & $\begin{array}{c}\text { Radial } \\
\text { width of new } \\
\text { unlignified } \\
\text { xylem }\end{array}$ \\
\hline & & number & $\mu$ & $\%$ of total & $\mu$ \\
\hline
\end{tabular}

TreE I

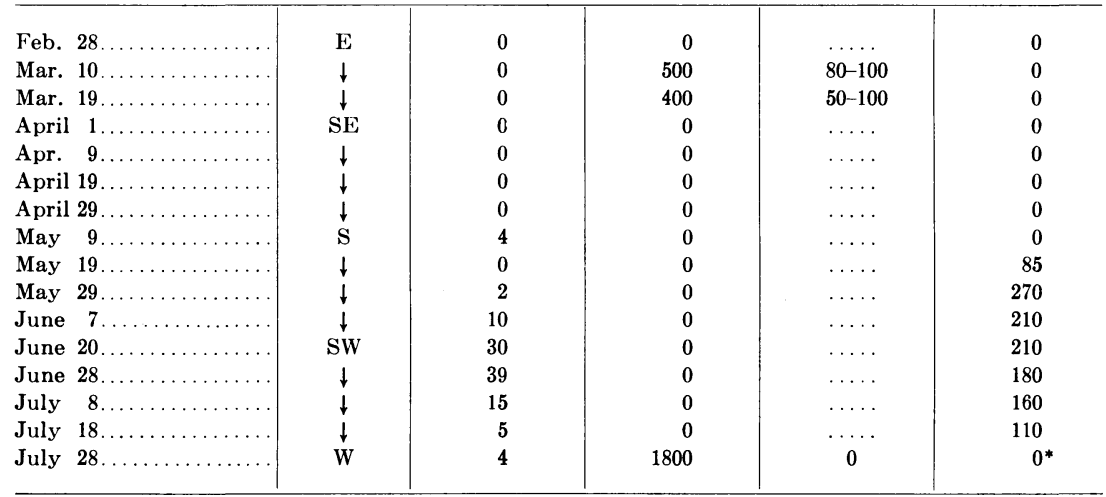

Tree II

\begin{tabular}{|c|c|c|c|c|c|}
\hline Aug. 7 . & $\mathbf{S}$ & 2 & 0 & $\ldots \ldots$ & 80 \\
\hline Aug. 18. & $\downarrow$ & 0 & 3500 & 0 & 160 \\
\hline Aug. 27. & $\downarrow$ & 0 & 200 & 0 & 160 \\
\hline Sept. 6... & SW & 0 & 1200 & 0 & 0 \\
\hline Sept. 16 . & $\downarrow$ & 0 & 0 & $\ldots \ldots$ & 0 \\
\hline Sept. 26. & $\downarrow$ & 0 & 0 & $\ldots \ldots$ & 0 \\
\hline Oct. $\quad 6 \ldots \ldots$ & W & 0 & 0 & $\ldots \ldots$ & 0 \\
\hline Oct. $16 \ldots \ldots$ & $\mathrm{E}$ & 7 & 2500 & $0-70$ & 40 \\
\hline Oct. $27 \ldots$ & $\downarrow$ & 2 & 0 & $\ldots \ldots$ & 160 \\
\hline Nov. $\quad 5 \ldots$. & $\downarrow$ & 0 & 1300 & $10-70$ & 110 \\
\hline Nov. $26 \ldots \ldots$. & SE & 3 & 900 & $20-40$ & 0 \\
\hline Dec. $24 \ldots \ldots$ & $\downarrow$ & 0 & 0 & $\ldots \ldots$ & 0 \\
\hline Jan. $26 \ldots \ldots$ & $\mathrm{S}$ & 0 & 0 & $\ldots \ldots$ & 0 \\
\hline
\end{tabular}

* Injury in part of the section was indicated by necrosis of sieve tubes. 
TABLE 1-Continued

Tree Pair IV

\begin{tabular}{|c|c|c|c|c|c|}
\hline \multirow[t]{2}{*}{$\begin{array}{l}\text { (From Feb. } 1947 \\
\text { to Jan. 1948) }\end{array}$} & \multirow[t]{2}{*}{$\begin{array}{l}\text { Side of tree } \\
\text { sampled }\end{array}$} & $\begin{array}{l}\text { Number of } \\
\text { slime bodies } \\
\text { present in } \\
4 \text { sections }\end{array}$ & $\begin{array}{c}\text { Length of } \\
\text { tangential } \\
\text { bands of } \\
\text { fiber initials }\end{array}$ & $\begin{array}{l}\text { Estimated } \\
\text { amount of } \\
\text { secondary wall } \\
\text { deposited on } \\
\text { fiber initials }\end{array}$ & $\begin{array}{c}\text { Radial } \\
\text { width of new } \\
\text { unlignified } \\
\text { xylem }\end{array}$ \\
\hline & & number & $\mu$ & $\%$ of total & $\mu$ \\
\hline
\end{tabular}

Tree I

\begin{tabular}{|c|c|c|c|c|c|}
\hline Feb. $28 \ldots$ & $\mathbf{E}$ & 0 & 0 & $\ldots \ldots$ & 0 \\
\hline Mar. $10 \ldots \ldots \ldots \ldots \ldots$ & $\downarrow$ & 0 & 0 & $\ldots \ldots$ & 0 \\
\hline Mar. $19 \ldots \ldots \ldots \ldots$ & $\downarrow$ & 0 & 0 & $\ldots \ldots$ & 0 \\
\hline April $1 \ldots \ldots$ & SE & 0 & 0 & $\ldots \ldots$ & 0 \\
\hline April $\mathbf{9} \ldots \ldots \ldots \ldots$ & $\downarrow$ & 0 & 0 & $\ldots \ldots$ & 0 \\
\hline April $19 \ldots$ & $\downarrow$ & 4 & 0 & $\ldots \ldots$ & 0 \\
\hline Apr. 29 . & $\downarrow$ & 0 & 0 & $\ldots \ldots$ & 0 \\
\hline May $\quad 9 \ldots \ldots \ldots$ & $\mathbf{S}$ & 3 & 0 & $\ldots \ldots$ & 0 \\
\hline May $19 \ldots$ & $\downarrow$ & 0 & 0 & $\ldots \ldots$ & 125 \\
\hline May 29. & $\downarrow$ & 0 & 0 & $\ldots \ldots$ & 320 \\
\hline June $7 \ldots$ & $\downarrow$ & 4 & 0 & $\ldots \ldots$ & 180 \\
\hline June $20 \ldots \ldots \ldots \ldots$ & SW & 42 & 0 & $\ldots \ldots$ & 180 \\
\hline June $28 \ldots \ldots \ldots \ldots$ & $\downarrow$ & 40 & 0 & $\ldots \ldots$ & 210 \\
\hline July $8 \ldots$ & $\downarrow$ & 23 & 0 & $\ldots \ldots$ & 180 \\
\hline July $18 \ldots \ldots \ldots \ldots$ & $\downarrow$ & 21 & 0 & $\ldots \ldots$ & 290 \\
\hline July $28 \ldots \ldots \ldots \ldots \ldots$ & W & 6 & 0 & $\ldots \ldots$ & 90 \\
\hline
\end{tabular}

Tree II

\begin{tabular}{|c|c|c|c|c|c|}
\hline Aug. $\quad 7 \ldots \ldots \ldots \ldots \ldots$ & $\mathrm{S}$ & 5 & 0 & $\ldots \ldots$ & 60 \\
\hline Aug. $18 \ldots \ldots \ldots \ldots \ldots$ & $\downarrow$ & 9 & 0 & $\ldots$ & 50 \\
\hline Aug. 27... & $\downarrow$ & 2 & 0 & $\ldots \ldots$ & 70 \\
\hline Sept. $6 \ldots \ldots \ldots \ldots \ldots$ & SW & 0 & 160 & $40-100$ & 75 \\
\hline Sept. $16 \ldots \ldots \ldots \ldots$ & $\downarrow$ & 0 & 0 & $\ldots \ldots$ & 0 \\
\hline Sept. $26 \ldots \ldots \ldots \ldots \ldots$ & $\downarrow$ & 0 & 0 & $\ldots$ & 0 \\
\hline Oct. $\quad 6 \ldots \ldots \ldots$ & W & 0 & 0 & $\ldots \ldots$ & 0 \\
\hline Oct. $16 \ldots \ldots$ & $\mathrm{E}$ & 1 & 2900 & 0-30 & 0 \\
\hline Oct. $27 \ldots \ldots \ldots \ldots \ldots$ & $\downarrow$ & 0 & 0 & $\ldots \ldots$ & 0 \\
\hline Nov. $\quad 5 \ldots \ldots \ldots \ldots \ldots$ & $\downarrow$ & 0 & 0 & $\ldots \ldots$ & $\mathbf{0}$ \\
\hline Nov. $26 \ldots$ & SE & 2 & 640 & $10-70$ & 0 \\
\hline Dec. $24 \ldots$ & $\downarrow$ & 0 & 900 & $40-70$ & 0 \\
\hline Jan. $26 \ldots$ & $\mathbf{S}$ & 0 & 600 & $30-70$ & 0 \\
\hline
\end{tabular}


TABLE 1-Continued

Tree Pair V

\begin{tabular}{c|c|c|c|c|c}
\hline \hline & $\begin{array}{c}\text { Number of } \\
\text { slime bodies } \\
\text { present in } \\
\text { 4 sections } \\
\text { to Jan. 1948) }\end{array}$ & $\begin{array}{c}\text { Length of } \\
\text { tangential } \\
\text { bands of } \\
\text { fiber initials }\end{array}$ & $\begin{array}{c}\text { Estimated } \\
\text { amount of } \\
\text { secondary wall } \\
\text { deposited on } \\
\text { fiber initials }\end{array}$ & $\begin{array}{c}\text { Radial } \\
\text { width of new } \\
\text { unlignified } \\
\text { xylem }\end{array}$ \\
\cline { 2 - 4 } & number & $\mu$ & \% of total & $\mu$ \\
\hline
\end{tabular}

Tree I

Feb. $28 \ldots \ldots \ldots \ldots \ldots \ldots$

Mar. 10.

Mar. 19.

April 1.

April 9

April 19.

April 29.

May 9

May 19 .

May 29.

June 7 .

June 20.

June 28 .

July 8 .

July 18 .

July 28 .

\begin{tabular}{|c|c|c|c|c|}
\hline $\mathbf{S}$ & 0 & 0 & $\ldots \ldots$ & 0 \\
\hline$\downarrow$ & 0 & 0 & $\ldots \ldots$ & 0 \\
\hline$\downarrow$ & 0 & 0 & $\ldots \ldots$ & 0 \\
\hline$\downarrow$ & 0 & 0 & $\ldots$ & 0 \\
\hline SW & 0 & 0 & $\ldots$ & 0 \\
\hline$\downarrow$ & 0 & 0 & $\ldots$ & 0 \\
\hline$\downarrow$ & 2 & 0 & $\ldots$ & 0 \\
\hline $\mathbf{W}$ & 2 & 0 & $\ldots \ldots$ & 0 \\
\hline $\mathbf{E}$ & 0 & 0 & $\ldots \ldots$ & 0 \\
\hline$\downarrow$ & 1 & 0 & $\ldots \ldots$ & 180 \\
\hline$\downarrow$ & 2 & 0 & $\ldots \ldots$ & 210 \\
\hline SE & 0 & 0 & $\ldots \ldots$ & 160 \\
\hline$\downarrow$ & 14 & 0 & $\ldots$ & 125 \\
\hline$\downarrow$ & 10 & 0 & $\ldots \ldots$ & 100 \\
\hline$\downarrow$ & 10 & 0 & $\ldots \ldots$ & 50 \\
\hline $\mathbf{S}$ & 10 & 0 & $\ldots \ldots$ & 50 \\
\hline
\end{tabular}

Tree II

\begin{tabular}{|c|c|c|c|c|c|}
\hline Aug. 7. & $\mathbf{E}$ & 7 & 0 & $\ldots \ldots$ & 120 \\
\hline Aug. 18 . & $\downarrow$ & 6 & 800 & 0 & 140 \\
\hline Aug. $27 \ldots \ldots$ & $\downarrow$ & 0 & 0 & $\ldots \ldots$ & 0 \\
\hline Sept. $6 \ldots \ldots$. & SE & 0 & 600 & 0 & 40 \\
\hline Sept. $16 \ldots \ldots$ & $\downarrow$ & 0 & 1500 & ó & 0 \\
\hline Sept. 26. & $\downarrow$ & 0 & 0 & $\ldots \ldots$ & 20 \\
\hline Oct. $\quad 6 \ldots \ldots \ldots \ldots$ & $\mathbf{S}$ & $\mathbf{0}$ & 0 & $\ldots$, & 90 \\
\hline Oct. $16 \ldots \ldots$ & $\downarrow$ & 1 & 0 & $\ldots \ldots$ & 30 \\
\hline Oct. $27 \ldots \ldots$ & $\downarrow$ & 0 & 0 & $\ldots \ldots$ & 120 \\
\hline Nov. 5.. & SW & 0 & 0 & $\ldots \ldots$ & 0 \\
\hline Nov. $26 \ldots . .$. & $\downarrow$ & 0 & 0 & $\ldots \ldots$ & $\mathbf{0}$ \\
\hline Dec. 24 . & $\downarrow$ & 0 & 0 & . & 0 \\
\hline Jan. 26.. & $\mathbf{W}$ & $\mathbf{0}$ & 0 & $\ldots \ldots$ & $\mathbf{0}$ \\
\hline
\end{tabular}


TABLE 1-Concluded

Average for Five Trees

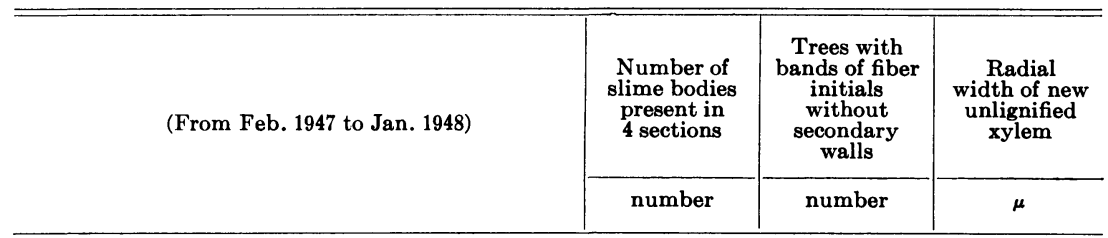

Tree I

Feb. 28 .

Mar. 10 .

Mar. 19.

April 1

April 9

April 19

April 29.

May 9.

May 19.

May 29

June 7 .

June 20.

June 28 .

July 8 .

July 18 .

July 28.

\begin{tabular}{r|r|r}
0 & 0 & 0 \\
0 & 0 & 0 \\
0 & 0 & 0 \\
0 & 0 & 0 \\
0 & 0 & 0 \\
0.8 & 0 & 0 \\
0.6 & 0 & 0 \\
2.2 & 0 & 0 \\
0.4 & 0 & 89 \\
0.8 & 0 & 228 \\
3.4 & 0 & 186 \\
20.6 & 0 & 184 \\
28.0 & 0 & 170 \\
16.8 & 1 & 162 \\
9.8 & 0 & 172 \\
6.0 & 3 & 94 \\
\hline
\end{tabular}

Tree II

\begin{tabular}{|c|c|c|c|}
\hline Aug. $\quad 7 \ldots \ldots \ldots \ldots \ldots \ldots \ldots$ & 3.8 & 2 & 96 \\
\hline Aug. $18 \ldots \ldots \ldots \ldots$ & 3.2 & 4 & 112 \\
\hline Aug. $27 \ldots$ & 0.5 & 0 & 77 \\
\hline 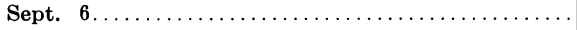 & 0 & 3 & 70 \\
\hline 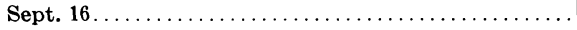 & 0.2 & 2 & 8 \\
\hline Sept. 26. . & 0.4 & $\mathbf{0}$ & 26 \\
\hline Oct. $\quad 6 \ldots \ldots \ldots \ldots$ & 0.6 & $\mathbf{0}$ & 44 \\
\hline 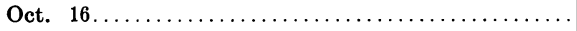 & 2.2 & 0 & 40 \\
\hline 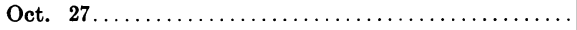 & 1.2 & 0 & 103 \\
\hline Nov. $5 \ldots$ & 1.4 & 0 & 59 \\
\hline Nov. $26 \ldots$ & 1.0 & $\mathbf{0}$ & 17 \\
\hline Dec. $24 \ldots$ & $\mathbf{0}$ & 0 & $\mathbf{0}$ \\
\hline 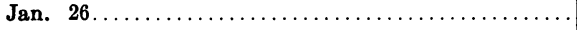 & $\mathbf{0}$ & 0 & 0 \\
\hline
\end{tabular}


changes in amount and character on July 28. Between July 18 and July 28 the temperature was over $105^{\circ} \mathrm{F}$ on five days. The humidity was low and the interval between irrigations was near its end (figure $1, C, D, E, F$ ). Xylem formation proceeded at a fair rate but slower than in the spring during the remainder of a cool August. September had three very hot periods of about five days each. On the last afternoon of a five-day hot spell (September 6), weeds were severely wilted but for this period the trees showed a marked increase in diameter, as indicated by measurements on the cool foggy morning of September 7. Xylem sections however indicated that growth was slowing down, and slippage did not occur in the cambium of three of five of the trees. On September 16 it was very hot and dry and near the end of a prolonged interval between irrigations. None of the sections had new xylem, the slippage of bark was not in the cambium of any of the trees, and trunk measurements indicated shrinkage. On September 26, following an irrigation, two trees showed renewed cambial activity. Tree 4 showed no further growth after September 6 and others showed no growth on the extreme west side.

\section{Periodicity of Secondary Xylem Production}

Before cambial activity began in the spring of the present experiment, the cambium was an indistinguishable part of a zone of undifferentiated cells. The adjoining xylem cell walls were lignified and had secondary thickenings (plate 1). Occasional small vessels with secondary walls protruded into the cambial zone (plate $2, B, C$ ). When the cambial cells at the center of the cambial zone became active in the spring the remainder of the undifferentiated cells on the xylem side of the cambium differentiated into terminal parenchyma. New xylem, formed during the early part of the grand period of growth, did not contain metatracheal parenchyma. The intertracheary tissue consisted entirely of fibers, crystal idioblasts and ray cells. Later, occasional patches of metatracheal parenchyma were formed, and in early August parenchyma was formed in more or less distinct bands. It is believed that banded metatracheal parenchyma followed growth stoppages.

In summary, each annual ring is composed of a wide ring of wood lacking metatracheal parenchyma cells, followed by wood with bands or patches of metatracheal parenchyma and ultimately by a band of terminal parenchyma. This last is interrupted by the rays and occasionally by small vessels (plate 3 ). Metatracheal bands may be interrupted by large vessels.

\section{Periodicity of Secondary Phloem Production}

Characteristics which can be used for detecting newly formed phloem are rather obscure as compared with those that distinguish the young from the old xylem (Esau, 1939). The presence of slime bodies in differentiating sieve tubes seems to be the most reliable indicator of phloem formation. Calcium oxalate crystals grow rapidly and may be completely formed and useless as indicators, whereas the slime bodies remain intact over a long enough period to be useful indicators (plate 4,C). Partially differentiated sieve tubes with thin cell walls are not a reliable character since thin walls may be found even in the winter when the cambium is dormant.

To determine the relative number of slime bodies present at various times 
of the year, four radial sections about 1 centimeter in length were mounted on a slide for each tree on each collection date. The number of slime bodies in each group of four radial sections is recorded in table 1 . It is seen that the slime-body stage of sieve-tube differentiation was not plentiful until after several weeks of xylem production. In 1947, when top growth started early, it was in June and July that most of the new phloem was produced. About July 28 sieve tube production gave way to fiber formation and several of the trees produced the first extensive bands of phloem fiber bundles observed for the season (plate $5, B$, and table 1 ). It apparently takes several weeks for the secondary walls to be deposited on the fibers; only fiber initials lacking secondary walls were observed from July 28 to September 16 (table 1). As the season progressed some bands of fibers continued to increase in length tangentially by the addition of more bundles of fiber initials at each end (plate $6, B)$. New shorter bands of bundles were also formed. As growth became sluggish at the end of the season the amount of secondary wall deposited on fibers of the same bundle differed widely (plate $6, C$ ). Some secondary wall formation for the 1946 season was not completed until the spring of 1947 (table 1). After radial growth slowed down about July 28, sieve tube production, as indicated by slime bodies, slowed down and became erratic until radial growth ceased in the autumn.

Hayward and Long (1942) report that bands of phloem fibers form the outermost portion of each growth zone in the secondary phloem of sweet orange and Valencia orange roots. For the trunks reported herein, most of the phloem was formed before fiber formation occurred, but some was also formed afterwards. There was no indication that fibers demarcated one season's growth from that of another. In fact, no direct way was found for determining how many years of phloem were present in a band of functioning phloem. Varying fiber patterns are shown in plate $5, A, B, C, D$.

\section{Secondary Phloem of the Sweet Orange}

Zones of the phloem. The secondary phloem of the sweet orange, and of other trees which have functioning phloem present throughout the year, may be separated into four zones characterized by stages of sieve tube maturation or degeneration in each. Esau (1939) has described the stages of sieve tube development and degeneration as they occur in the higher plants. Some of the zones not previously named are here given names and characterized so that they may be conveniently referred to. In the orange bark there is next to the cambium at certain times of the year a narrow zone of phloem with the sieve tubes in the developmental stage. The tissue containing the developing sieve tubes will be referred to here as the developing phloem. Adjoining the developing phloem is a relatively wide band of phloem with the sieve tubes in the mature stage. 'This zone is commonly referred to as the functioning phloem (Esau, 1950). Adjoining the functioning phloem on the outer margin is a narrow zone in which the sieve tubes are in the transitional and the degenerative stages. Definitive callus forms on the sieve plates and the sieve tubes lose their turgor (transitional stage). The callus is then dissolved from the sieve plates, the cytoplasm and other contents in the sieve tubes are dissolved away and the sieve tubes are flattened by pressure from adjacent pa- 
renchyma cells (degenerative stage). The narrow zone containing sieve tubes in these two stages will be referred to as the degenerating phloem. Outside the degenerating phloem is a vast area containing degenerated sieve tubes (the ultimate of the degenerative stage). This area is commonly called the nonfunctioning phloem.

The developing phloem. Cells of the developing phloem are formed by tangential divisions of the cambium, and those from which the vertical system is formed are fusiform in shape like the cambial cells (plate $7, B$ ). The fusiform derivatives destined to become sieve tube elements undergo divisions by radial walls, which divide them into young companion cells and young sieve tubes (plate $4, B$ ). The sieve tube elements frequently develop in groups of two to several in radial rows, and the tangential walls between them remain thin compared to other tangential walls (plate $4, A, B$ ). Perhaps there is a division of the cambial derivative by a tangential wall which precedes the division of the cells by radial walls. The companion cells and not the sieve tubes adjoin the ray cells whenever sieve tube companion-cell units are adjacent to the rays. Slime bodies occur in the young sieve tubes as they enlarge. In the present experiment they were usually cylindrical in shape and usually void of vacuoles in the fixed material described (plate $4, A, C, D$ ). Apparently walls of sieve tubes undergoing expansion are frail because they are usually folded and wavy in outline after fixing and sectioning (plate $6, A$ ), whereas the walls of the mature sieve tubes remain rigid. Slime bodies and nuclei of the sieve tubes degenerate upon maturation.

Phloem parenchyma arise by the formation of three transverse walls across fusiform mother cells (plate 7, $C$ ). Other fusiform mother cells were divided transversely into as many as 25 crystal idioblasts (plate $4, C$ ). In addition to the transverse division to form the crystal idioblasts from phloem mother cells, there may also occasionally be a radial division. Crystal-bearing cells are frequently located near fiber bundles. Phloem fibers are formed by elongation of cambial derivatives, and, as previously stated, their formation occurs in the greatest numbers at the end of an active period of phloem production in early summer.

The functioning phloem. In the trunks of sweet orange trees there is adjacent to the developing phloem a band of functioning phloem of approximately $1 / 2$ to $1 \mathrm{~mm}$ thickness which, as indicated by the cytological condition of the sieve tubes, is in functioning condition at all times of the year (plates $1 ; 5 ; 7, A, C)$. This part of the phloem has been described by Webber and Fawcett (1935) and a summary of their work has been presented in the review of literature. The existence of bands of parenchyma alternating with bands of sieve tubes, as observed by these workers, has not been observed in the present work. Bands of fibers interrupted only by the ray cells may be absent or present in numbers varying from one to several in the functioning phloem (plate 5).

The sieve tubes are irregular in outline as seen in cross section. Their sizes are also quite variable depending on the age and horticultural variety of the tree. Nacré walls such as are found in sieve tubes of many other plants are lacking. Contrary to the report of Webber and Fawcett (1935), sieve plates are oblique (plate 8) and rarely, if ever, horizontal. As many as six sieve 
areas occur in the sieve plates (plate 8) and callus is frequently not detected on functioning sieve areas. Sieve tubes are formed by the sieve elements joining other sieve tube elements above and below them, which may be slightly to one side of the other in tangential direction but not in radial direction.

The degenerating phloem. Sieve tubes and companion cells of the outer margin of the functioning phloem gradually degenerate. Callus forms on the sieve plates and then the sieve tubes and companion cells lose their turgor and collapse. Eventually they are stretched tangentially and flattened by increase in girth of the tree. In some sections, however, areas of nonfunctioning phloem were observed which showed little or no flattening. During this degenerative phase the callus and cytoplasmic contents are removed. Many of the parenchyma cells are also flattened and lose their contents. Sieve tubes undergoing obliteration at any one time are few in number and form only a fringe between the functioning and the nonfunctioning crushed phloem. The callusing of sieve plates at the outer margin of the functioning phloem showed no seasonal variations in abundance. No method was devised for determining when sieve tube degeneration occurs at the greatest rate.

The nonfunctioning phloem. The nonfunctioning phloem assumes the functions of cortical tissue and constitutes a large part of the bark of the sweet orange tree trunk. The trunk bark consists of the developing, functioning, degenerating and nonfunctioning zones of the phloem and of the periderm. Considerable changes occur in the nonfunctioning phloem after its formation by the degeneration of the functioning phloem. The ray cells are stretched tangentially and divide by radial walls to form masses of parenchymatous tissue in which groups of sclereids may form (plate 1). Between these blocks of tissues derived from the rays, the vertical system in the old phloem tissue is further compacted until the lumina of the cells are completely obliterated. As a result, only masses of folded, flattened walls remain. The bands of fiber groups remain intact and are brought closer together as a result of crushing of the nonfunctioning phloem.

\section{DISCUSSION}

It is generally agreed that there are three distinct periods of shoot extension growth in orange trees in southern California. On the other hand, although root extension growth has been found to alternate with top growth in young trees (Crider, 1927 ; Waynick and Walker, 1930 ; Reed and McDougal, 1937), the present work and that of others indicate that root growth overlaps shoot. growth in older trees and is almost continuous except when temperatures are limiting.

The current investigations confirm others that trunk measurements are not always reliable for detecting the radial growth which has occurred over short periods of time. The presence of new xylem mother cells and of slime bodies in sieve tubes seems to be the best indicator of recent growth. The occurrence of free slippage at least partly through the initiating layer of cambial cells and the remainder through the xylem mother cells, with no tearing of the cambial zone from the face of the phloem or maturing xylem, seems to be the best indicator of current growth.

Radial growth in orange trees in some respects was parallel to that found 
for ring-porous trees by Lodewick (1928). He found that there was a period of spring wood production which ended with a rest period in cambial activity and the appearance of the first phloem fibers and stone cells. In the present work with orange trees, it was found that early wood lacked metatracheal parenchyma but that some was gradually formed. About July 28 extensive bands of phloem fibers were observed and there was a growth stoppage in some trees. On August 7 metatracheal parenchyma was present in bands. It is believed that bands of metatracheal parenchyma may be formed after growth stoppages in much the same way that terminal parenchyma is formed from the innermost cells of the cambial zone when growth is resumed in the spring. In both the ring-porous hard woods studied by Lodewick and the orange trees, radial growth was much slower after the first rest period.

There is circumstantial evidence that a combination of maximum temperature over $100^{\circ} \mathrm{F}$ and increased water tension at the ends of irrigation periods brought about radial growth stoppages on two occasions. On the other hand the normal amount of vessels formed in connection with new leaves may have been completed on the two growth stoppage dates and there may have been no relation to external factors. Shoot and root growth were not affected by these adverse periods.

No direct evidence was obtained in the present study on the age of the oldest sieve tubes in the functioning phloem. Circumstantial evidence indicates that the functioning phloem present was more than two years old. Since the orange tree leaves stay on the tree about two years, one might suspect that the sieve tubes which differentiated in connection with them would function for about two years. It is seen in table 1 that there was a period of vigorous sieve tube production in late June and July, as is indicated by the presence of slime bodies, and that there was practically no fiber production. Then in late July, August, and early September there was a period of fiber production with reduced sieve tube production. After September 16, there were no fiber bands seen without at least part of the secondary walls laid down, and sieve tube production was occurring slowly. Possibly it took three months for laying down secondary walls. If this sequence were consistent, one would expect to find a wide band of phloem and an area of fibers for each year's phloem, and plate $5, C$ and $D$, might be taken as showing three increments and in addition part of a fourth increment of phloem. However, fiber production is so erratic that such a conclusion is somewhat speculative. Plate $5, C$ and $D$, also suggest that the phloem formed during the main period of phloem production (June and July) is limited to the narrow band between the cambium and the lowermost fiber bands. The latter are mature and must have been formed the year before.

\section{SUMMARY}

1. The time at which new trunk phloem was produced, with respect to the time of shoot and root extension growth and xylem production, was investigated for the trunks of 30-year-old sweet orange trees during 1947. Also considered were the anatomic features of xylem and phloem development, the portion of the phloem which was replaced during the year, and how and when the older sieve tubes degenerated. 
2. There were three distinct flushes of top growth.

3. Root growth periods completely overlapped the second and third shoot growth flushes but there was one distinct rest period.

4. When the cambium was active, slippage occurred partly in the row of initiating cells and partly in the xylem mother cells. When the cambium was inactive and slippage occurred it was either at the face of the xylem or phloem or through the cambial zone or in xylem mother cells.

5. Cambial activity began in the trunk about three months after top growth began.

6. New xylem was evident 20 or 30 days before the slime body stage of sieve tube development became abundant.

7. During the early or grand period of growth, xylem production proceeded at a rapid uniform rate and metatracheal parenchyma was rare.

8. Phloem production also occurred the most abundantly during the grand period of growth during which fiber production was rare.

9. On July 28 cambial activity had ceased temporarily in some trees, extensive bands of phloem fibers were present in the phloem, and on August 7 and August 18 extensive bands of metatracheal parenchyma were present which are believed to have been a result of the growth stoppage.

10. Growth after July 28 was erratic. On some collection dates the cambium was active on some trees in some positions and not active on other trees. The amount of unlignified xylem was variable.

11. A second rest period, observed on September 6 and 16, may have been related to a hot dry period at the end of an interval between irrigations.

12. The annual ring of the xylem had some fairly definite characteristics. There was a row of terminal parenchyma which contained only small vessels. Then there was a wide region of "spring" wood which lacked metatracheal parenchyma, especially at the beginning of the season. Wood produced after the rest period terminating the spring growth contained rows of metatracheal parenchyma. The bands of metatracheal parenchyma differed from the terminal parenchyma by being traversed by large vessels.

13. For the detection of phloem production, the presence of slime bodies in developing sieve tubes was found to be the most useful indicator.

14. There was no way of detecting annual rings in the functioning phloem but several rings are hypothesized to have occurred. During the grand period of growth most of the year's phloem was produced. Following this, fiber initials were observed in sections from several of the trees, and during the remainder of the season a little more phloem and possibly a few more fibers were formed.

15. For convenience in referring to various regions of the phloem, names (some of them new) have been applied to them. They are the developing phloem, the functioning phloem, the degenerating phloem, and the nonfunctioning phloem. Brief descriptions are given for each from the developmental point of view. The number of sieve tubes observed to be in the degenerating stage was about the same throughout the year but this does not indicate the velocity of degeneration.

16. A detailed method for collecting, sectioning, and staining large batches of citrus phloem is presented in the appendix. 


\section{ACKNOWLEDGMENTS}

The author is indebted to Mr. Oscar F. Clarke for preparing the slides and to Dr. Katherine Esau and Dr. F. Murray Scott for reading and making suggestions for improving the manuscript. 


\section{LITERATURE CITED}

Caladan, E. C.

1947. Shell bark of lemons. Calif. Citrog. 32:232, 233, 263-65.

CAMERON, S. H., and C. A. SCHROEDER

1945. Cambial activity and starch in bearing orange trees. Amer. Soc. Hort. Sci. 46:55-59.

CHOWDHURY, K. A., and K. N. TANDAN

1950. Extension and radial growth in trees. Nature 165:732-33.

CRIDER, F. J.

1927. Root growth of citrus trees with practical applications. Citrus Leaves 7:1-3, 27-30.

DAUBENMIRe, R. F., and M. E. Deters

1947. Comparative studies of growth in deciduous and evergreen trees. Bot. Gaz. $109: 1-12$.

EsAu, KATHERINE

1939. Development and structure of the phloem tissue. Bot. Rev. 5:373-432.

1948. Phloem structure in the grapevine, and its seasonal changes. Hilgardia 18:217-96. 1950. Development and structure of the phloem tissue. II. Bot. Rev. 16:67-114.

HAASIS, F. W.

1934. Diametral changes in tree trunks. Carnegie Inst. Wash. Pub. 450:1-103.

HAYW ARD, H. E., and E. M. LONG

1942. The anatomy of the seedling and roots of the Valencia orange. U. S. Dept. Agr. Tech. Bul. 786:1-31.

LODEWICK, J. ELTON

1928. Seasonal activity of the cambium in some northeastern trees. N. Y. State Col. Forestry Bul. 1(2-a) :1-87. Tech. Pub. 23.

MacDougal, D. T.

1936. Studies in tree-growth by the dendrographic method. Carnegie Inst. Wash. Pub. $462: 1-256$.

Priestlex, J. H.

1930. Studies in the physiology of cambial activity. III. The seasonal activity of the cambium. New Phytol. 29:316-54.

1935. Radial growth and extension growth in the tree. Forestry 9:84-95.

REED, H. S., and D. T. MACDougaL

1937. Periodicity in the growth of the orange tree. Growth 1:371-73.

SCHNEIDER, HENRY

1946. Histological studies. Part III in progress report on quick decline studies. Calif. Citrog. 31:198-99.

W ALlaCE, J. M., and H. S. FAWCETT

1947. Quick decline of orange trees; a virus disease. Science 105:315-16.

WEBBer, IRMA E., and H. S. FAWCETT

1935. Comparative histology of healthy and psorosis-affected tissues of Citrus sinensis. Hilgardia 9 : 71-93.

WAYNICK, D. D., and S. J. WALKER

1930. Rooting habits of citrus trees. California Citrogr. 15:201. 


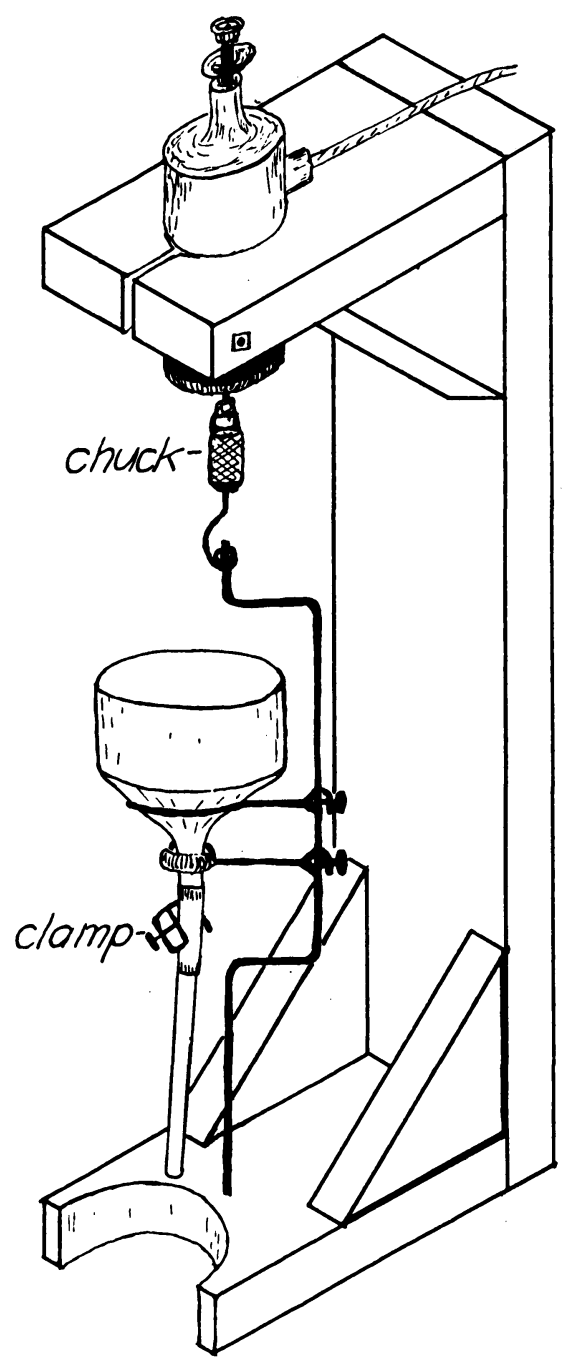

Appendix fig. 1. Diagrammatic drawing of the staining apparatus.

\section{APPENDIX: COLLECTING AND STAINING BARK SPECIMENS}

There has been a demand for methods of collecting and staining large quantities of bark specimens in connection with studies on seasonal changes in the phloem, diagnosing orange trees for quick decline, and in studying the relationship of sieve-tube necrosis in lemon trees to lemon tree decline. These methods are here presented in detail.

The bark and wood were removed by driving a rectangular-shaped die into the tree. Samples trimmed into small pieces were then placed in Randolph's Modified Navashin's solution for 24 to 48 hours. Radial and transverse sections 16 microns thick were cut on a sliding microtome with the aid of a freez- 
ing attachment. Sections were placed in staining baskets made by fusing nickel wire cloth (50 mesh for large sections, 100 mesh for small) to one end of a piece of glass tubing ( $20 \mathrm{~mm}$ diameter, $35 \mathrm{~mm}$ high $)$. The staining baskets were placed in a Büchner funnel on a ring stand which could be rotated from the top end by a variable speed mixing motor (figure 1). The motor was equipped with a chuck. A $1 / 16$ inch diameter wire connected the chuck to the metal rod supporting the funnel in such a way that it rotated freely on the rod and was slightly off center from the end of the wire that was in the chuck. A clamp on a short piece of rubber tubing attached to the stem of the funnel was handy for quick changing of staining solutions and wash water. The rotating motion of the funnel caused the sections to swirl about in the stains and hence to stain evenly. By this method as many as 42 batches of material were stained simultaneously without the tedious work of decanting.

Sections were mordanted in 4 per cent ferric ammonium sulfate for from 4 to 20 hours, washed several times in distilled water, stained progressively with dilute Heidenhain's haematoxylin (one ec of a stock solution of $21 / 2$ per cent haematoxylin in 95 per cent ethyl alcohol, in 500 cc of slightly alkaline water) until the cell walls were gray. They were then washed several times in water and stained for 15 hours to several days in $1 / 4$ per cent lacmoid. They were then rinsed briefly in several changes of tap water and placed in 1 per cent sodium bicarbonate for 5 minutes or longer. Several baskets at a time were then removed from the funnel of sodium bicarbonate solution and dehydrated in 30 per cent, 70 per cent, and several changes of 95 per cent alcohol (about 1 minute in each), cleared in two changes of a good grade of clove oil (straw colored), and mounted in neutral Canada Balsam. Between changes, adhering solutions were removed from the baskets by blotting on paper towels. Fading will occur if sections are mounted near the edges of cover slips. Fading can be retarded by dusting the balsam at the edges of cover slips with sodium bicarbonate. Lignified cell walls and callus on sieve plates were stained blue and other structures various shades of gray. 



\section{PLATES}





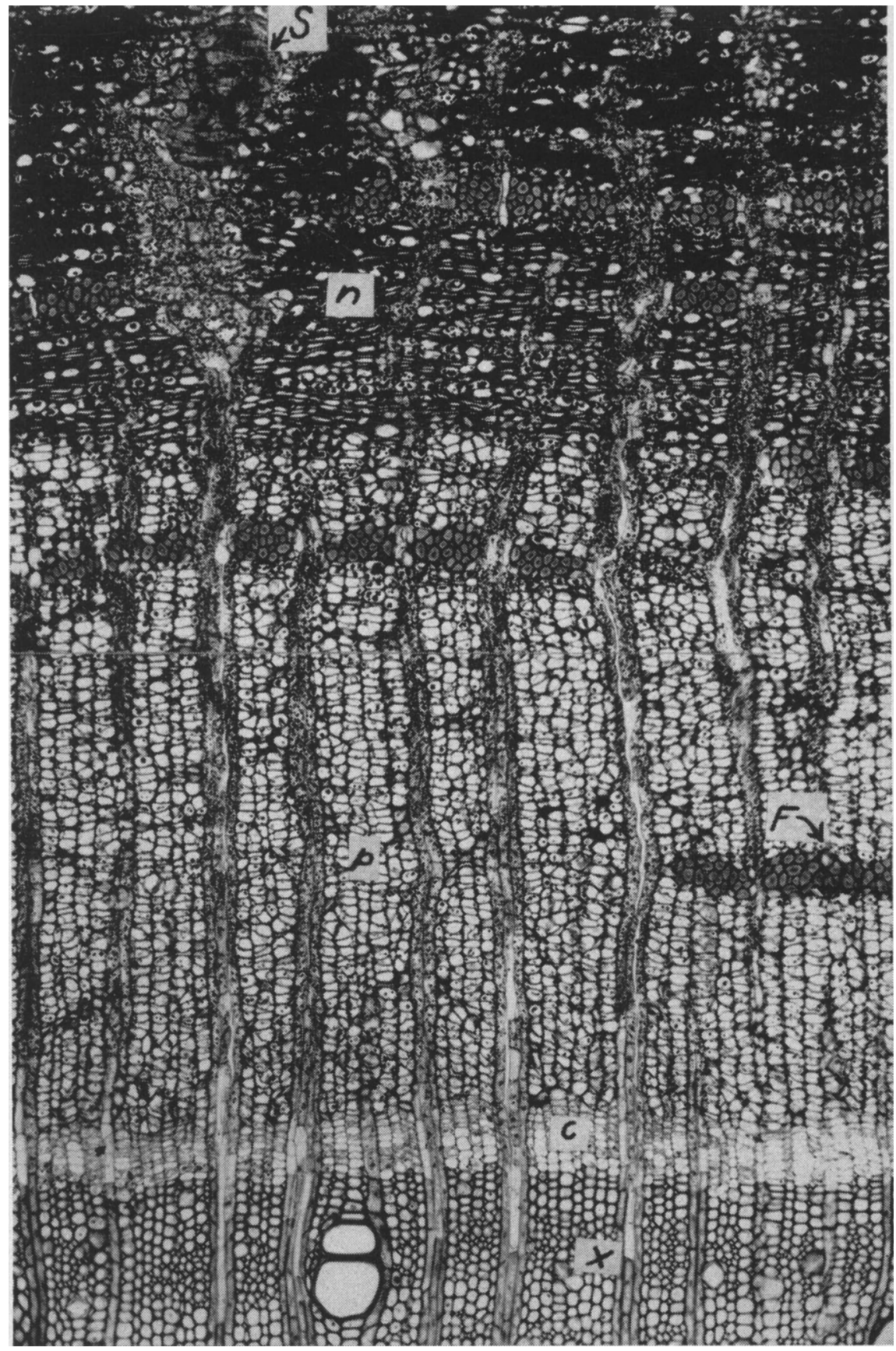

Plate 1. Transverse section of portion of trunk collected April 29, 1947, showing a small portion of xylem $(x)$, the cambial zone $(c)$, the functioning phloem $(P)$, and part of the nonfunctioning phloem $(n)$. One band of phloem fibers is shown at $F$, and a group of sclereids at $S .(\times 120$. 
Plate 2. Transverse sections of the cambial region during dormancy $(A, B, C)$, at the beginning of cambial activity $(D)$, and after cambial activity had progressed $(E)$. Collection dates: $A, B$, April $9 ; C, D$, April $29 ; E$, August 18. In $A$ the bark slipped from the wood at the face of the xylem (X), through the cambial zone (C), and at the face of the phloem (P). In $B$ slippage was primarily at the face of the phloem and the cambial zone adhered to the wood. In $C$ only slight slippage occurred at the xylem. In $D$ the cambium was becoming active where shown, but at other parts of the section the bark and wood were still together. In $E$ slippage occurred both at the cambium (C) and in the differentiating xylem (D). (All $\times 120$. 

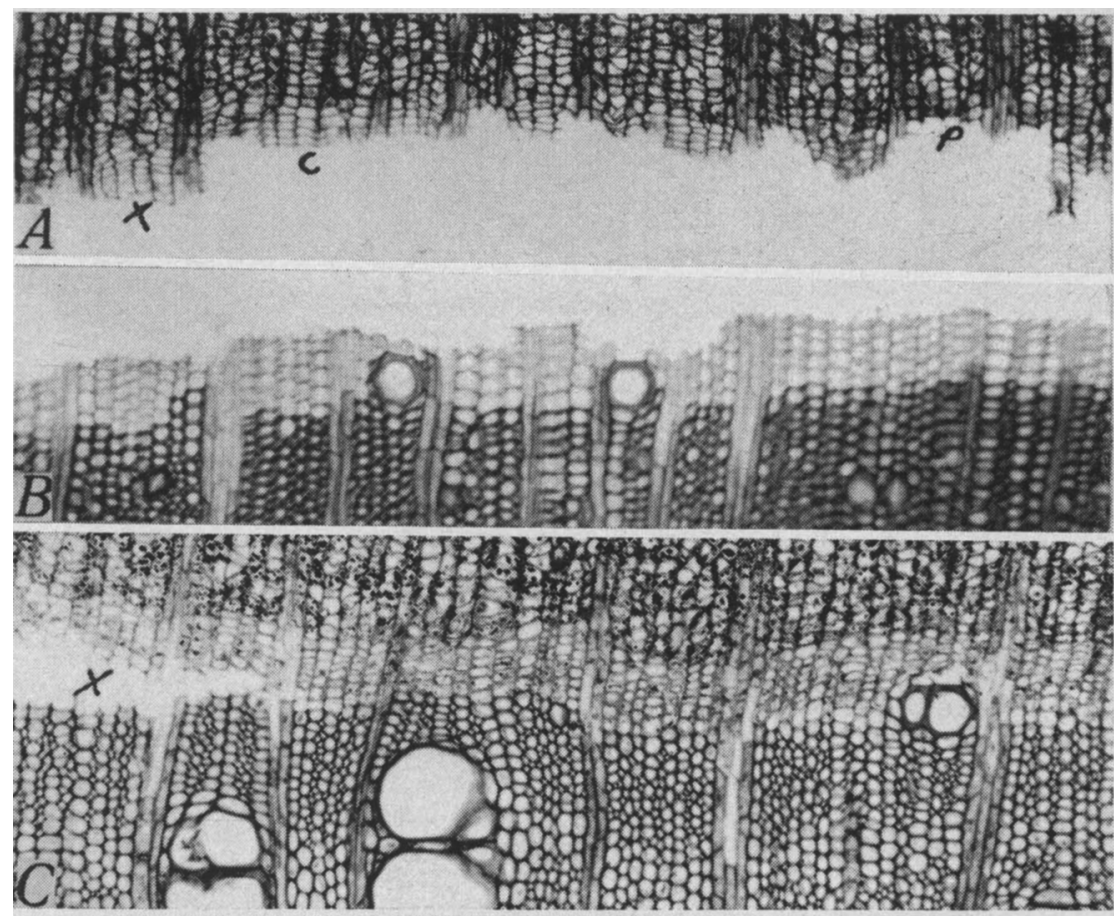
7.
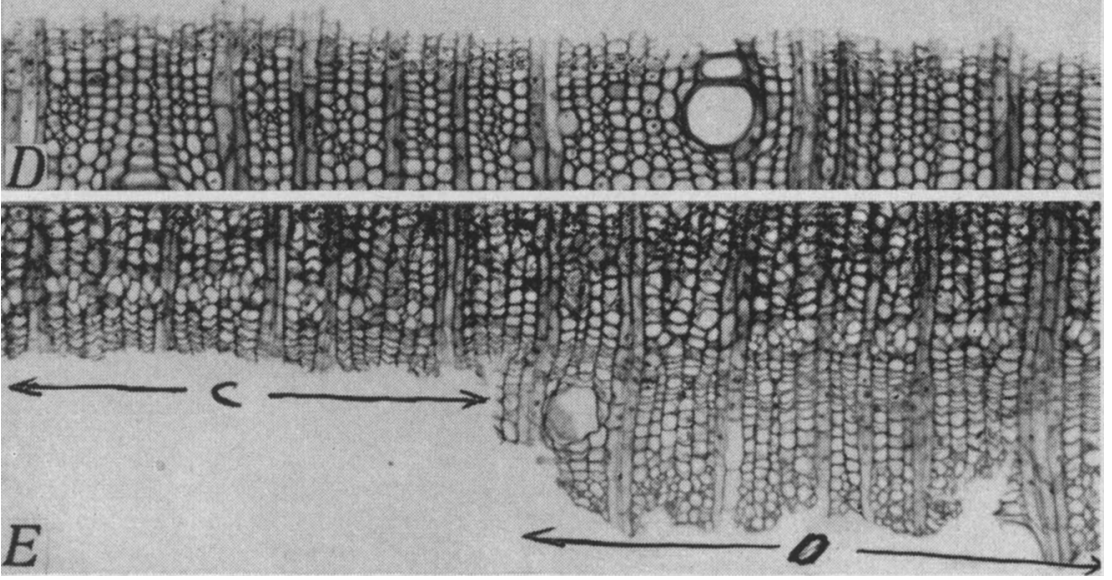


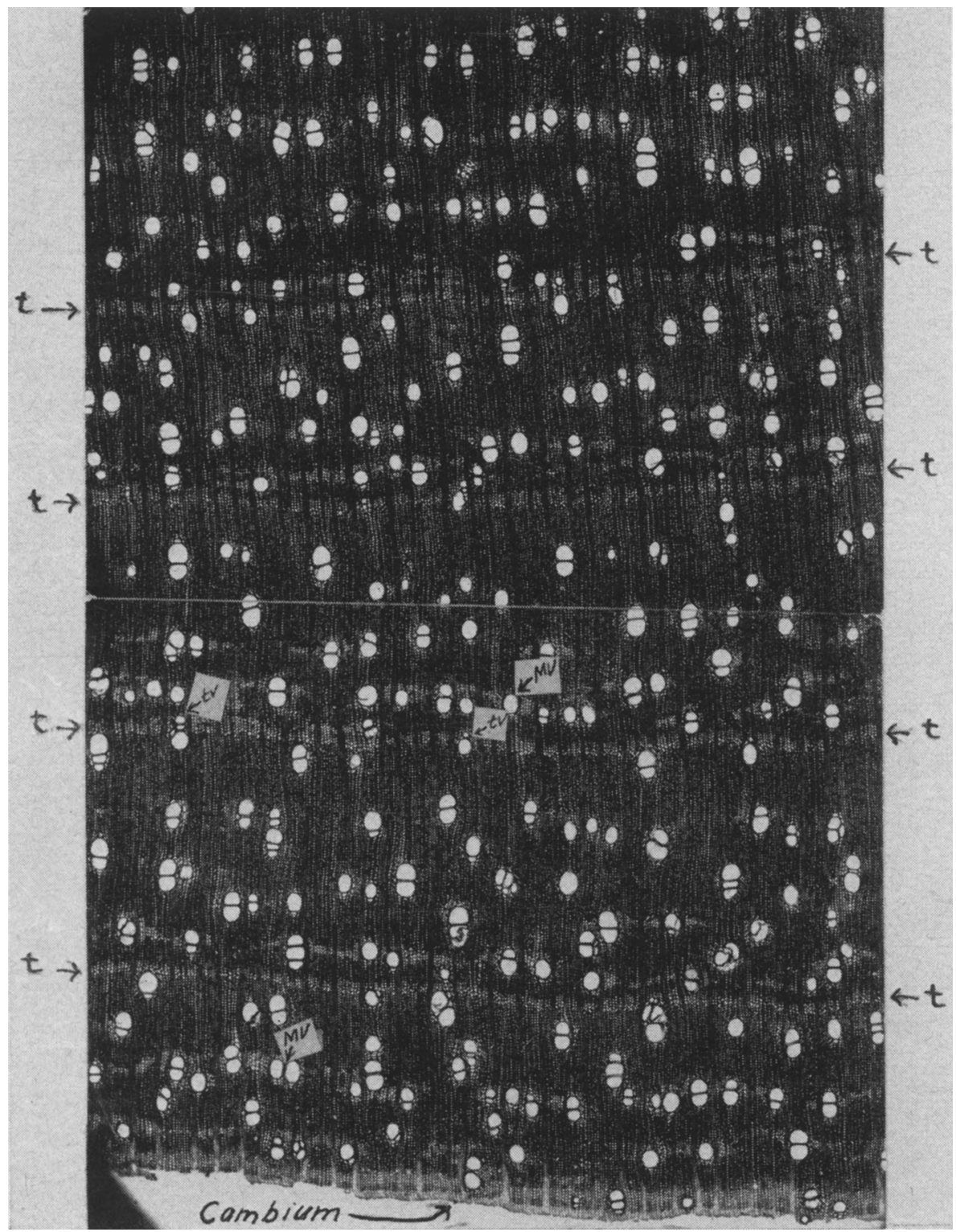

Plate 3. Cross section of the xylem showing possibly five annual rings. The first wood produced each year lacks metatracheal parenchyma. Vessels are only occasionally found in the terminal parenchyma $(t)$ and when they are, they are usually small (tv), whereas those found in metatracheal parenchyma may be large $(\mathrm{mv})$. Collected October $7 .(\times 24$. $)$ 

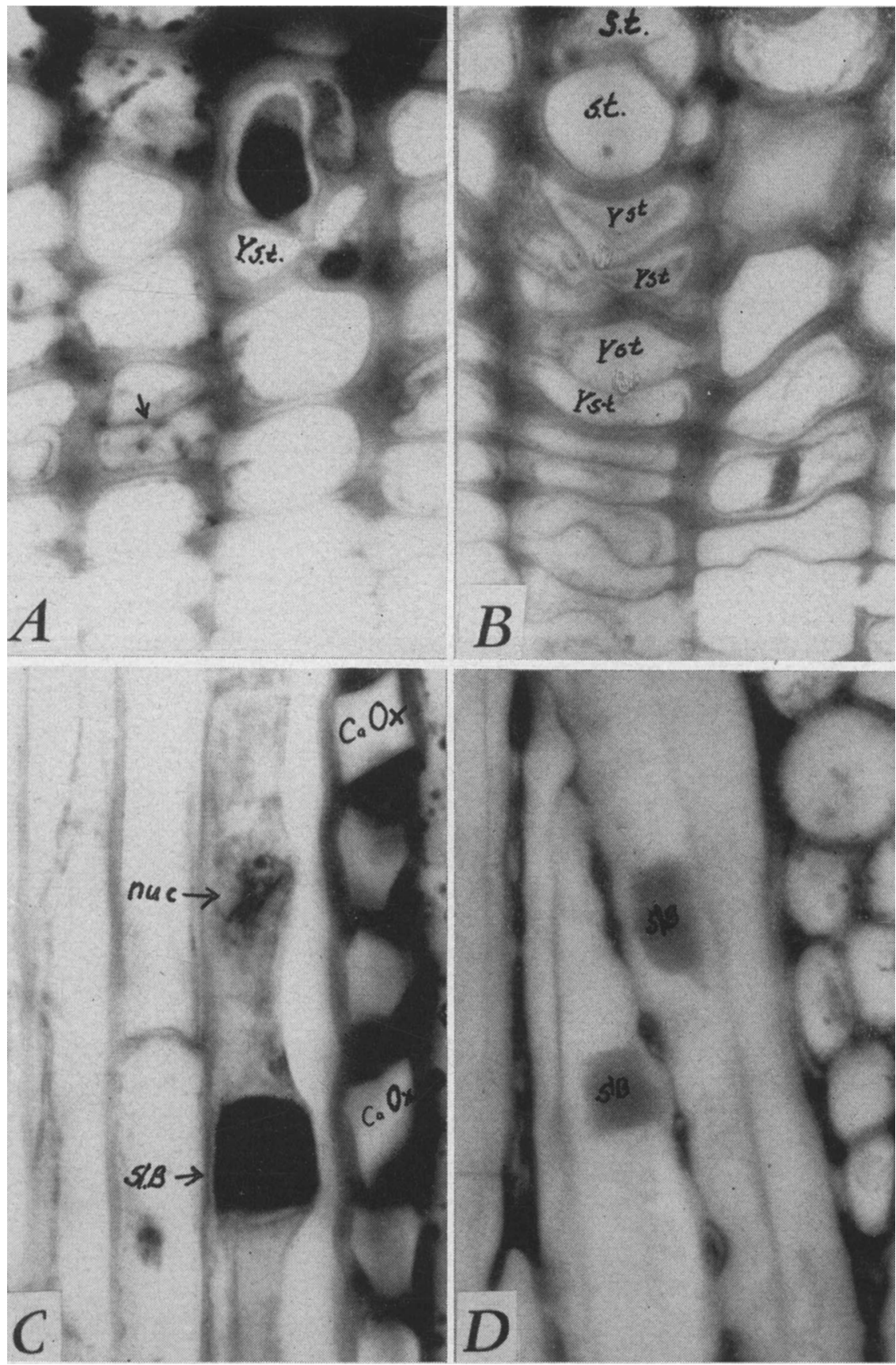

Plate 4. Transverse sections $(A, B)$, radial section $(C)$, and tangential section $(D)$ of developing phloem. Thin walls occur between the young sieve tubes ( $Y$ st). Slime bodies (Sl B) may be seen in $A, C$, and $D$. Calcium oxalate erystals $(\mathrm{CaOx})$ in crystal cells may be seen in C. $(A, \times 1400 ; B, C, D, \times 1200$. $)$ 

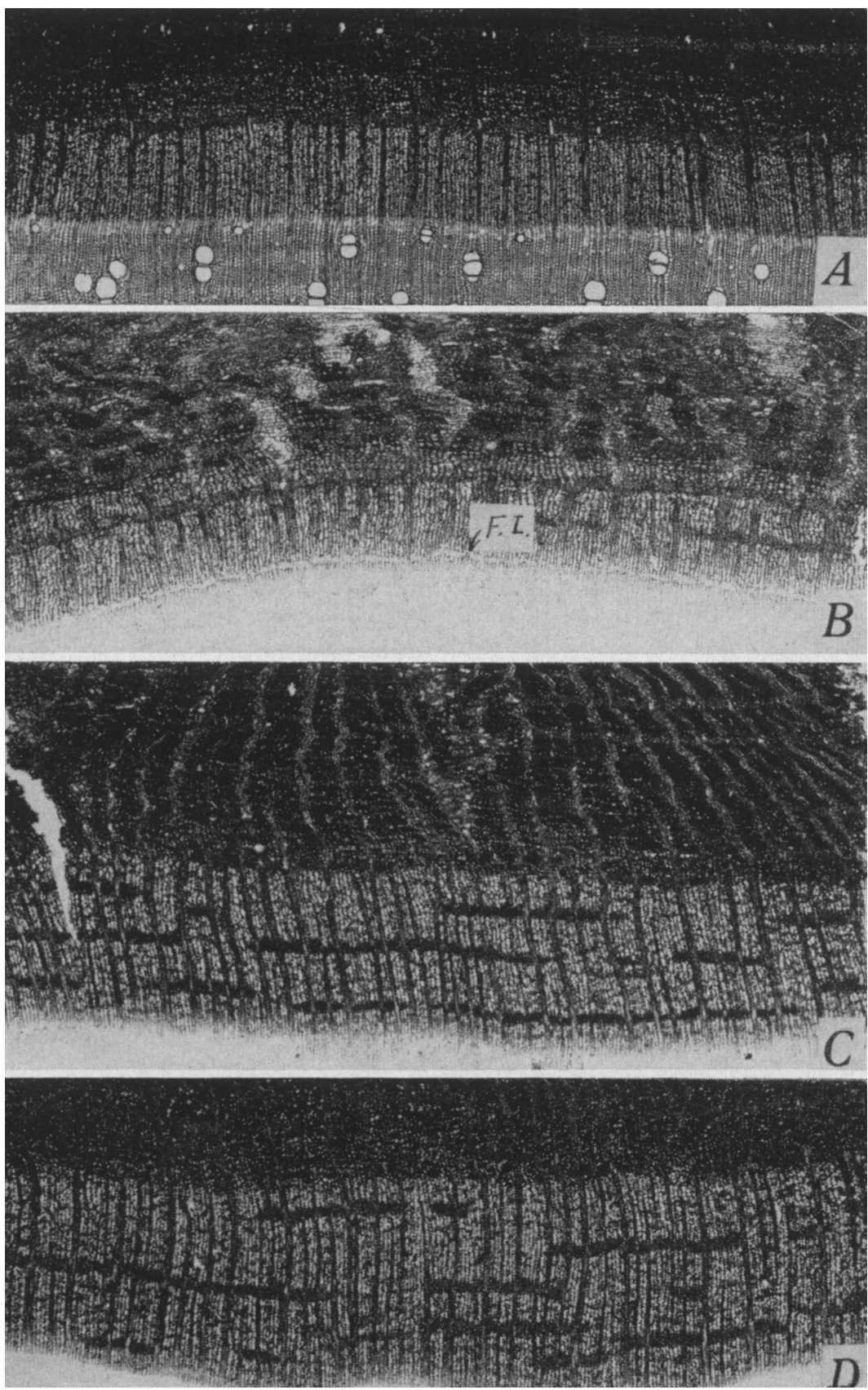

Plate 5. Transverse sections showing the variability in production of fiber bands in the phloem. Fibers in the functioning phloem are lacking $(A)$, numerous and uniform $(B, C)$, or numerous and irregular $(D)$. A band of fiber initials (F.I.) is shown in $B$. Note that the bark has torn from the wood through the xylem mother cells in $B$. ( $A$, collected April 19; $C$, July $18 ; B, D$, July 28 .) (All $\times 28$.) 
Plate 6 (page 364). Transverse sections of the developing phloem. $A$, the developing sieve tubes (d) at the top of the plate are flattened and have wavy walls. (Collected June 28.) $B$, a band of fiber initials formed over a period of time by the addition of younger bundles on the left. (Collected October 16.) $C$, fibers within the same bundles are shown with varying degrees of wall formation indicating very slow phloem production. (Collected November 5.) $(A, \times 1400 ; B, \times 260 ; C, \times 435$. $)$ 

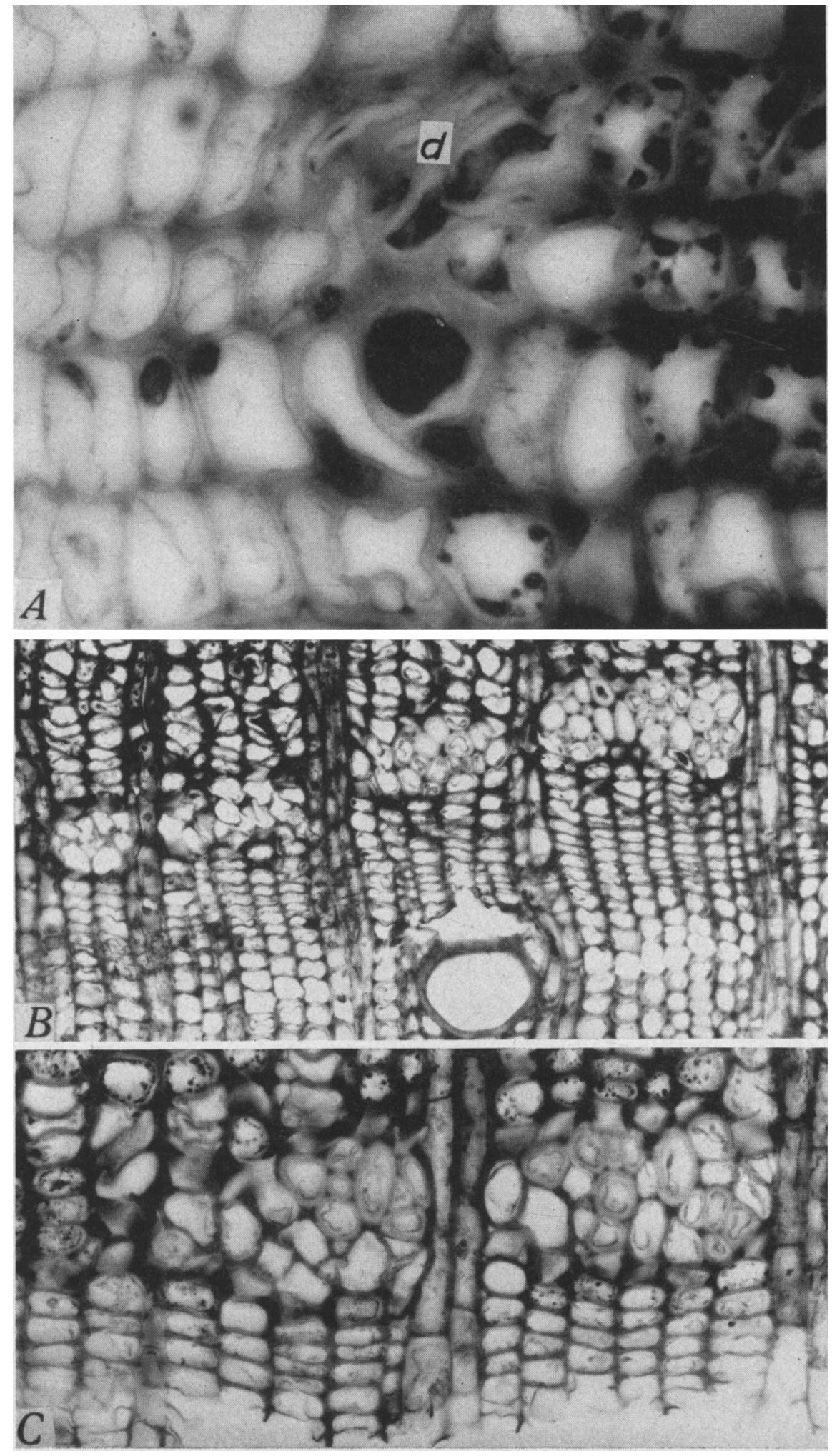


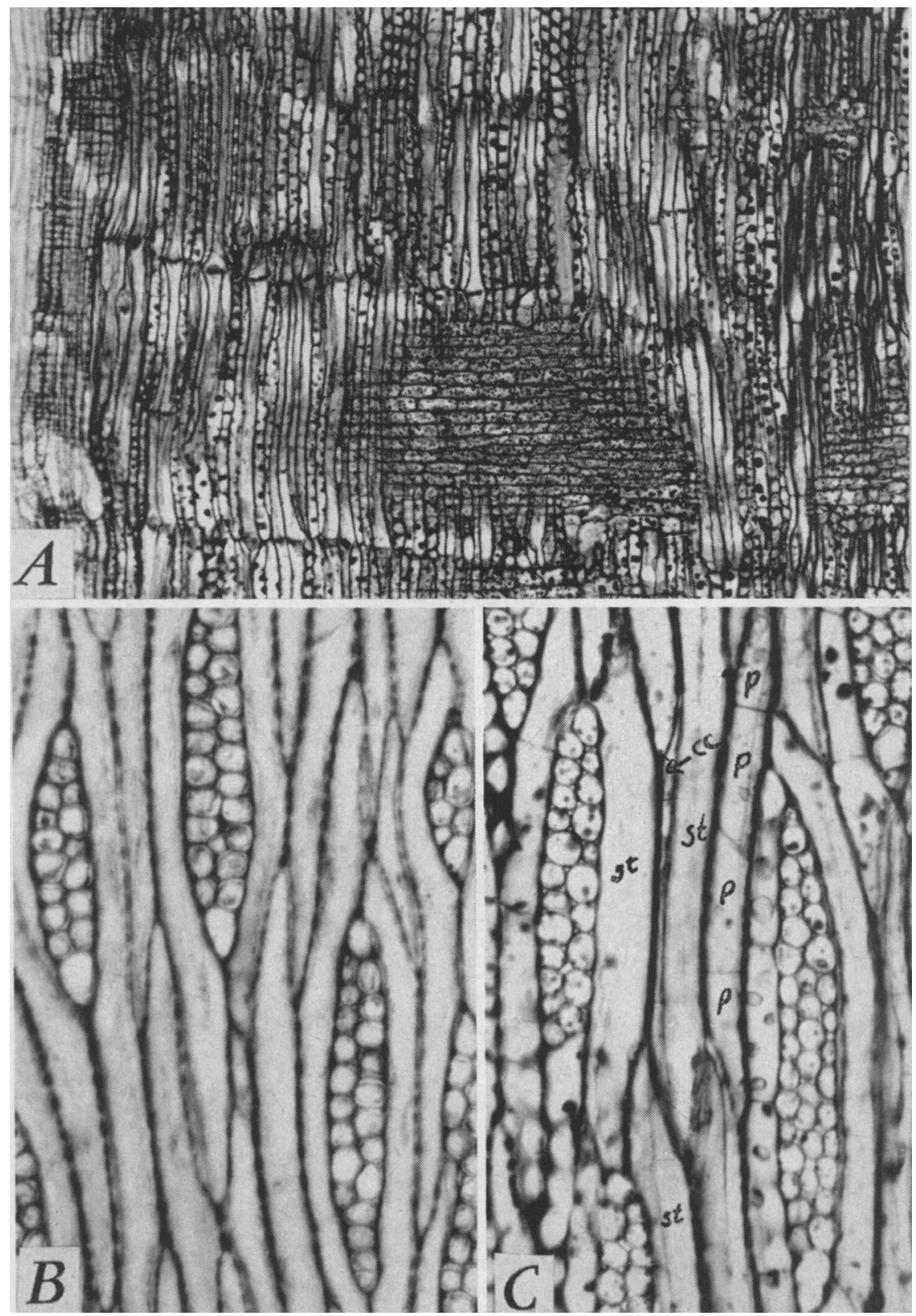

Plate 7. Radial view $(A)$ and tangential view $(C)$ of the phloem, and tangential view $(B)$ of the cambium of bark collected April 19. In $A$ there is a narrow zone of wood on the left and then the cambium. The functioning phloem occupies the center of the picture and a narrow strip of nonfunctioning phloem is on the right. Ray parenchyma, erystal bearing cells, and sieve tubes may be seen. In $B$ the fusiform and cambial ray cells are shown. The fusiform cells have pitted walls. In $C$ the sieve tubes (st), companion cells (ec), parenchyma cells $(\mathrm{P})$, crystal bearing cells, and rays are shown. $(A, \times 120 ; B, C, \times 300$. $)$ 

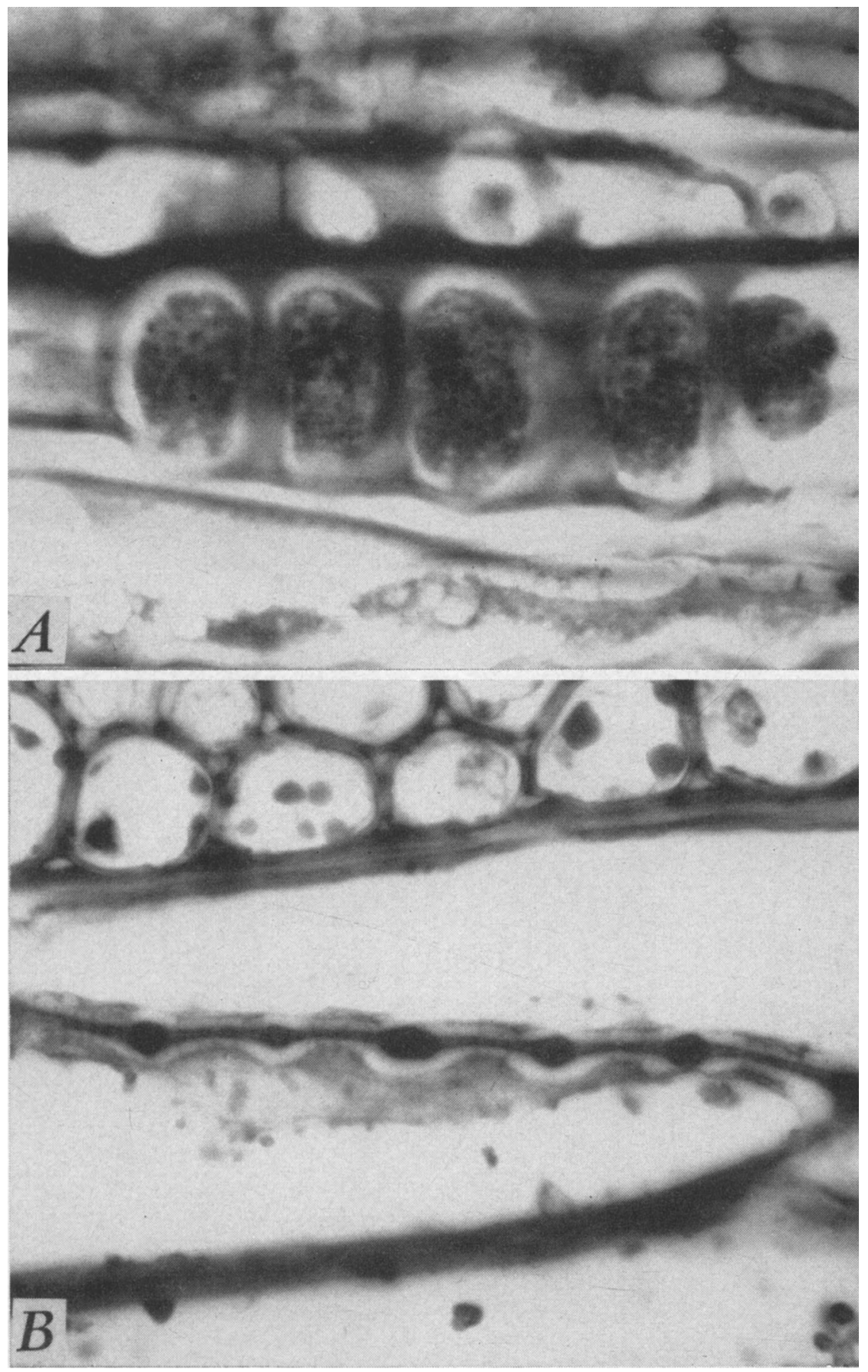

Plate 8. Radial $(A)$ and tangential section $(B)$ of mature sieve plates showing the sieve areas. (Both $\times 1700$.) 
The journal Hilgardia is published at irregular intervals, in volumes of about 600 pages. The number of issues per volume varies.

Subscriptions are not sold. The periodical is sent as published only to libraries, or to institutions in foreign countries having publications to offer in exchange.

You may obtain a single copy of any issue free, as long as the supply lasts; please request by volume and issue number from:

Publications Office

\section{College of Agriculture}

Berkeley 4, California

The limit to nonresidents of California is 10 separate issues on a single order. A list of the issues still available will be sent on request. 


\section{CONTENTS}

Introduction $\ldots \ldots \ldots \ldots \ldots \ldots \ldots \ldots \ldots \ldots \ldots \ldots \ldots \ldots \ldots \ldots \ldots \ldots \ldots$

Review of Literature $\ldots \ldots \ldots \ldots \ldots \ldots \ldots \ldots \ldots \ldots \ldots \ldots \ldots \ldots \ldots \ldots \ldots$

Materials and Methods ............................ 334

Observations during $1947 \ldots \ldots \ldots \ldots \ldots \ldots \ldots \ldots \ldots \ldots \ldots \ldots \ldots \ldots \ldots \ldots \ldots \ldots \ldots$

Periodicty of extension growth of shoots and roots and radial growth of

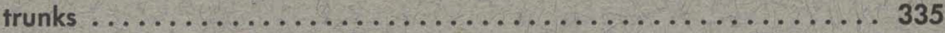

Periodicity of secondary xylem production $\ldots \ldots \ldots \ldots \ldots \ldots \ldots . . \ldots \ldots$

Periodicity of secondary phloem production $\ldots \ldots \ldots \ldots \ldots \ldots \ldots 344$

Secondary phloem of the sweet orange $\ldots \ldots \ldots \ldots \ldots \ldots \ldots \ldots .345$

Discussion ................................ 347

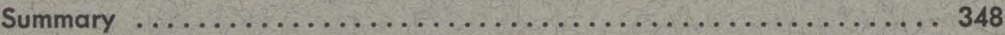

Appendix: Collecting and Staining Bark Specimens ............ 352

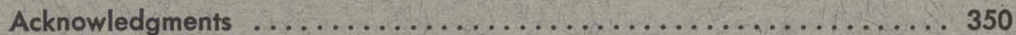

Literature Cited $\ldots \ldots \ldots \ldots \ldots \ldots \ldots \ldots \ldots \ldots \ldots \ldots \ldots \ldots \ldots \ldots \ldots$ 